\title{
I9. COMMISSION DE LA VARIATION DES LATITUDES
}

\section{President: M. KImura, Director of the International Latitude Observatory.}

Membres: MM. Aguilar, G. Bemporad, Benes, Bianchi, Burton, Carnera, de Grama, Dodwell, Esclangon, Hartmann, Hashimoto, J. Jackson, Jones, W. D. Lambert. Littell, Muller, Nijland, Perez, Schlesinger, Schumann, Stetson, Subbotin, Voute, Williams.

\section{A. Provisional Results of the Observations of the Latitude Variation} DURING THE PASt Three Years

\section{International Latitude Service in the North Parallel.}

The values given below are those published in each annual report of the international latitude work. They were calculated from the observations at five stations, except the last part of I934 which was made without Kitab, because the observation books from Kitab since November 1934 have arrived too late at the Central Bureau.

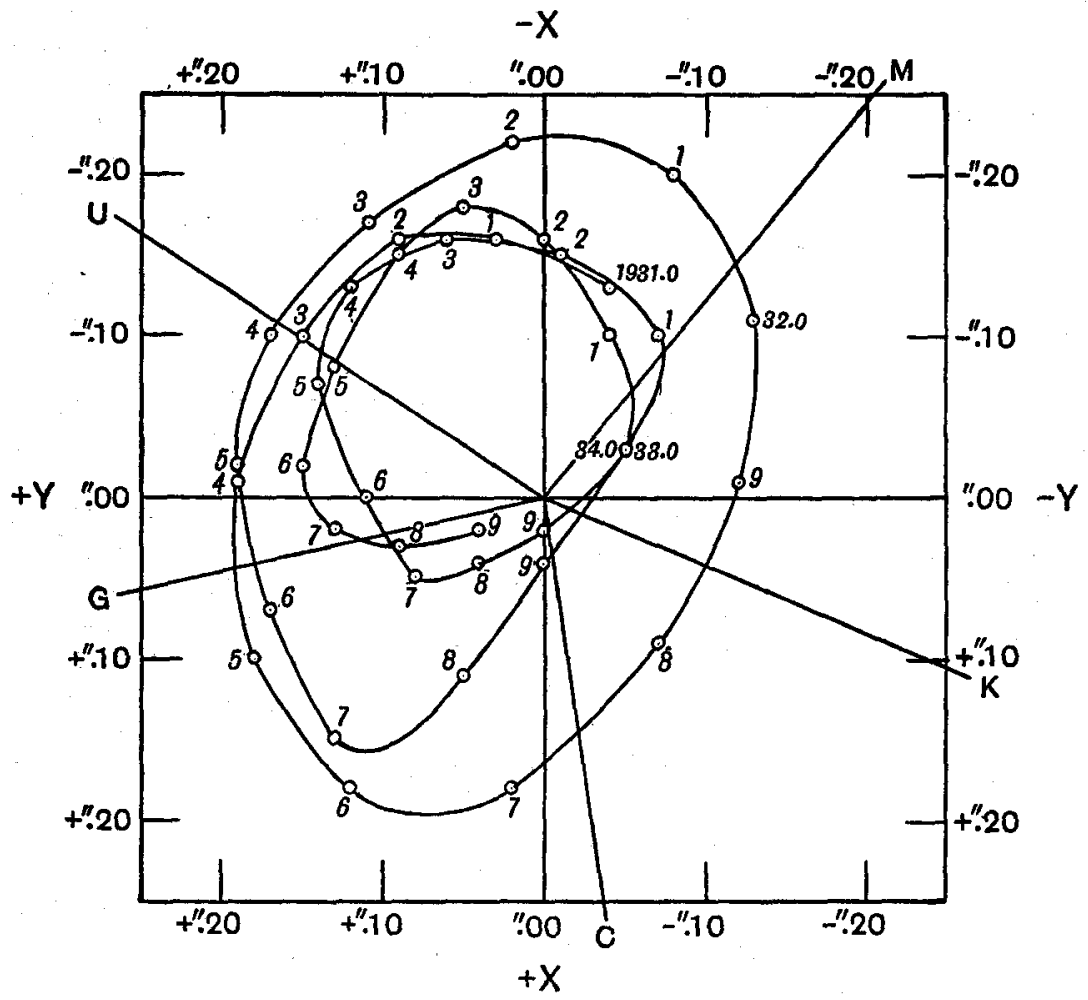

Path of the Polar Motion, 1931.0-34.9. 


\begin{tabular}{|c|c|c|c|c|c|c|}
\hline \multirow[b]{3}{*}{$\begin{array}{c}\text { Fraction } \\
\text { of year }\end{array}$} & \multicolumn{6}{|c|}{ Year } \\
\hline & \multicolumn{2}{|c|}{1932} & \multicolumn{2}{|c|}{1933} & \multicolumn{2}{|c|}{1934} \\
\hline & $X$ & $Y$ & $X$ & $Y$ & $\underset{ }{X}$ & $Y$ \\
\hline $\begin{array}{l}.0 \\
.1 \\
.2 \\
.3 \\
.4 \\
.5 \\
.6 \\
.7 \\
.8 \\
.9\end{array}$ & $\begin{array}{l}-.11 \\
-.20 \\
-.22 \\
-.17 \\
-.10 \\
-.02 \\
+.07 \\
+.15 \\
+.11 \\
+.04\end{array}$ & $\begin{array}{r}-\cdot 13 \\
-.08 \\
+\cdot 02 \\
+\cdot 11 \\
+\cdot 17 \\
+\cdot 19 \\
+\cdot 17 \\
+\cdot 13 \\
+.05 \\
.00\end{array}$ & $\begin{array}{r}-.03 \\
-.10 \\
-.15 \\
-.16 \\
-.13 \\
-.07 \\
.00 \\
+.05 \\
+.04 \\
+.02\end{array}$ & $\begin{array}{r}-.05 \\
-.07 \\
-.01 \\
+.06 \\
+.12 \\
+.14 \\
+.11 \\
+.08 \\
+.04 \\
.00\end{array}$ & $\begin{array}{l}-.03 \\
-.10 \\
-.16 \\
-.18 \\
-.15 \\
-.08 \\
-.02 \\
+.02 \\
+.03 \\
+.02\end{array}$ & $\begin{array}{r}-.05 \\
-.04 \\
.00 \\
+.05 \\
+.09 \\
+.13 \\
+.15 \\
+.13 \\
+.09 \\
+.04\end{array}$ \\
\hline
\end{tabular}

2. Royal Observatory, Greenwich.

The following values were taken directly from M.N. No. 5, March of each year.

\begin{tabular}{|c|c|c|c|}
\hline \multirow[b]{2}{*}{$\begin{array}{l}\text { Fraction } \\
\text { of year }\end{array}$} & \multicolumn{3}{|c|}{ Year } \\
\hline & 1931 & $\underset{\text { " }}{1932}$ & $\begin{array}{c}1933 \\
"\end{array}$ \\
\hline $\begin{array}{l}\cdot 0 \\
.1 \\
.2 \\
.3 \\
.4 \\
.5 \\
.6 \\
.7 \\
.8 \\
.9\end{array}$ & $\begin{array}{l}-.16 \\
-.22 \\
-.20 \\
-.14 \\
-.06 \\
+.03 \\
+.12 \\
+.17 \\
+.04 \\
-.05\end{array}$ & $\begin{array}{l}-.12 \\
-.19 \\
-.21 \\
-.19 \\
-.15 \\
-.05 \\
+.12 \\
+.13 \\
+.08 \\
+.02\end{array}$ & $\begin{array}{r}-.05 \\
-.12 \\
-.18 \\
-.20 \\
-.18 \\
-.08 \\
+.03 \\
+.10 \\
+.09 \\
+.03\end{array}$ \\
\hline
\end{tabular}

3. Naval Observatory, Washington.

The following values were taken from $A . J$. Nos. 973, I000, IoI9.

\begin{tabular}{cccc} 
& \multicolumn{3}{c}{ Year } \\
\cline { 2 - 4 } $\begin{array}{c}\text { Fraction } \\
\text { of year }\end{array}$ & 1931 & 1932 & 1933 \\
.0 & -.14 & -.17 & - \\
.1 & -.10 & -.15 & -.20 \\
.1 & -.03 & -.12 & -.13 \\
.2 & +.02 & -.04 & -.11 \\
.3 & +.05 & +.01 & -.06 \\
.4 & +.01 & +.04 & -.01 \\
.5 & -.03 & +.04 & +.01 \\
.6 & -.09 & +.01 & +.04 \\
.7 & -.16 & -.08 & +.01 \\
.8 & -.18 & -.15 & -.05 \\
.9 & & &
\end{tabular}




\section{Z for Greenwich and Washington.}

The following $z$ for each observatory is obtained by taking the simple annual means of residuals in the sense Greenwich or Washington minus International for each tenth of the year, during the period of three years I93I-33.

$\begin{array}{ccc}\begin{array}{c}\text { Fraction } \\ \text { of year }\end{array} & \overbrace{z_{g}}^{2031-33} \\ .0 & -.020 & -.077 \\ .1 & -.023 & -.073 \\ .2 & -.020 & -.087 \\ .3 & -.033 & -.113 \\ .4 & -.050 & -.137 \\ .5 & -.037 & -.153 \\ .6 & +.007 & -.143 \\ .7 & +.007 & -.117 \\ .8 & -.010 & -.100 \\ .9 & -.017 & -.090\end{array}$

The above values of $z$ except the constant parts have very similar runs with the corresponding ones reported in Transactions of I.A.U. 4, I932. Such facts can be seen very clearly by looking at the following results of the harmonic analysis according to the annual and semi-annual parts.

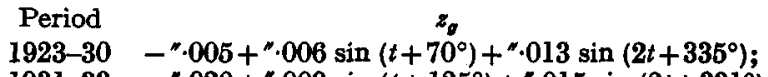

$$
\begin{aligned}
& 1931-33-" .020+" .009 \sin \left(t+125^{\circ}\right)+" .015 \sin \left(2 t+331^{\circ}\right) \text {. } \\
& \begin{array}{c}
z_{v} \\
1923-30-.046+" .034 \sin \left(t+82^{\circ}\right)+.013 \sin \left(2 t+253^{\circ}\right) ; \\
1931-33 \quad-.0109+.037 \sin \left(t+81^{\circ}\right)+.006 \sin \left(2 t+298^{\circ}\right)
\end{array}
\end{aligned}
$$

It is very interesting to note that the peculiarities for both observatories, viz. the constancy of the semi-annual part at Greenwich and that of the annual one at Washington, hold continuously through the whole of their own series; but that their constant parts are increasing numerically pretty considerably in both observatories while their signs remain unchanged.

\section{South International Station at Adelaide.}

The following values of the variation of latitude at this station were directly

\begin{tabular}{|c|c|c|c|}
\hline \multirow[b]{2}{*}{$\begin{array}{l}\text { Fraction } \\
\text { of year }\end{array}$} & \multicolumn{3}{|c|}{ Year } \\
\hline & 1931 & 1932 & 1933 \\
\hline $\begin{array}{l}.06 \\
.14 \\
.22 \\
.30 \\
.39 \\
.47 \\
.56 \\
.64 \\
.72 \\
.80 \\
.89 \\
.97\end{array}$ & $\begin{array}{l}= \\
= \\
= \\
= \\
-.24 \\
=.25 \\
=.13 \\
-.02 \\
+.22\end{array}$ & $\begin{array}{l}+\cdot 30 \\
+\cdot 30 \\
+\cdot 27 \\
+\cdot 13 \\
+\cdot 07 \\
-\cdot 25 \\
-.20 \\
-.29 \\
-.31 \\
-.19 \\
-.05 \\
+\cdot 15\end{array}$ & $\begin{array}{l}+.10 \\
+.13 \\
+.12 \\
+.08 \\
+.02 \\
-.02 \\
-.06 \\
-.08 \\
-.10 \\
-.07 \\
-.02 \\
+.03\end{array}$ \\
\hline
\end{tabular}
taken from the annual reports. 
From the residuals substracted by the latitude variation of the polar motion, we got the following $z_{a}$ for the two different periods:

$$
\begin{array}{ll}
\text { I932.06-32.97 } & z=+ \text { ".012 +".09 } \sin \left(2 \odot-\alpha-46^{\circ}\right) \\
\text { I933.06-33.97 } & z=+" \cdot 025+" .07 \sin \left(2 \odot-\alpha+48^{\circ}\right) .
\end{array}
$$

\section{South International Station at La Plata.}

At this station the observations complete in all respects, as in the other international stations, were begun in August r934 under the direction of the new Director, Mr Aguilar. Thus there is no material to report here, until one complete year passes.

\section{Batavia International Latitude Station.}

The detailed report with minute and interesting discussions on the results of observations during the period I931.73-33.97 was published by the Topographic Service at Batavia in Jaarverslag van den Topografischen Dienst in NederlandschIndië over I933.

The following observed values of the variation of latitude were taken directly

\begin{tabular}{|c|c|c|c|c|c|c|}
\hline Group & Date & {$\left[\Delta \phi_{m}\right]$} & Date & $\left.\underset{n}{\left[\Delta \phi_{m}\right.}\right]$ & Date & {$\left[\Delta \phi_{m}\right]$} \\
\hline IV & & & 1932.08 & +.321 & 1933.03 & $+\cdot 525$ \\
\hline $\mathrm{V}$ & & & .15 & $+\cdot 309$ & .12 & +.515 \\
\hline VI & & & .23 & +.225 & .21 & $+\cdot 488$ \\
\hline VII & & & .31 & +.097 & .31 & $+\cdot 384$ \\
\hline VIII & & & .39 & +.065 & .39 & $\begin{array}{r}+291 \\
\end{array}$ \\
\hline IX & & & .47 & -.021 & .48 & +.312 \\
\hline $\mathbf{x}$ & & & .56 & +.019 & .55 & +.325 \\
\hline XI & & & .65 & $+\cdot 106$ & .63 & $+\cdot 385$ \\
\hline XII & & & .74 & +.250 & .72 & $+\cdot 484$ \\
\hline I & 1931.8 & +.081 & .81 & $+\cdot 378$ & .80 & $+\cdot 564$ \\
\hline II & .8 & $+\cdot 133$ & .88 & +.450 & .87 & $+\cdot 701$ \\
\hline III & .9 & +.229 & .96 & $+\cdot 539$ & .97 & +856 \\
\hline
\end{tabular}
from those given on $\mathrm{p}$. ro of the above cited report.

The above series was formed by the successive summation of the difference between two consecutive values of the same group obtained by its evening and morning observations. Therefore, as seen in the table, the constant part of $z$ which may be due to the local diurnal variation of latitude is becoming larger and larger with the time. It is noteworthy that the sense of this variation is opposite to those of the same kind in the north international stations. This change of sign may, however, be easily explained by an appropriate choice of the phase angle of a diurnal form $-a_{1} \cos \left(\odot-\alpha+A_{1}\right)$; assuming $a_{1}=$ ". Io, as I have done usually, $A_{1}$ will be $-27^{\circ}$.

In addition the Batavia report has given the interesting results of an investigation into the residuals of observations after subtracting the pure polar motion deduced from the provisional international results and also by the linear variation above quoted. The residuals were solved in two ways, firstly as only a function of the Chandler Period and secondly as that of an annual period, with better results for the assumption of an annual period than for the other. Thus the existence of the local annual term of $z$ being affirmed, I have tried to find the amplitude and the phase of the second term of $z$, namely $+a_{2} \sin \left(2 \odot-\alpha+A_{2}\right)$, not from the residuals 
given in the Batavian report but from the direct value of $\Delta \phi_{m}$ (see p. Io of the report) corrected by the variation of latitude of the pure polar motion. Thus I have obtained $+" \cdot 039 \sin \left(2 \odot-\alpha-16^{\circ}\right)$.

Here it ought to be noted that the three observations in the year I93I have been omitted in my calculation. As a matter of fact, the observations for the first three or four groups are in wide disagreement with any computed ones, while the whole remainder of the series agrees very closely with them. In my experience, however, such discrepancies might take place pretty often especially in the case of the start of new observations. They may probably be due to the unsettled state of the foundation and of the instrument, and moreover the observer himself is not familiarized with every thing regarding the observations even though he is skilful and experienced. According to the circumstances, such an unstable state may even continue for about half a year.

8. R. Osservatorio di Milano (Italy).

Latitude observations during the years I929, I930, I93I, I932.

\begin{tabular}{|c|c|c|c|c|c|c|c|c|}
\hline \multirow[b]{3}{*}{$\begin{array}{l}\text { Fraction } \\
\text { of year }\end{array}$} & \multicolumn{8}{|c|}{ Year } \\
\hline & \multicolumn{2}{|c|}{1929} & \multicolumn{2}{|c|}{1930} & \multicolumn{2}{|c|}{1931} & \multicolumn{2}{|c|}{1932} \\
\hline & $+45^{\circ} 41^{\prime}$ & $\mathrm{O}-\mathrm{C}$ & $+45^{\circ} 41^{\prime}$ & $\mathrm{O}-\mathrm{C}$ & $+45^{\circ} 41^{\prime}$ & $\mathrm{O}-\mathrm{C}$ & $+45^{\circ} 41^{\prime}$ & O-C \\
\hline $\begin{array}{l}\cdot 0 \\
.1 \\
.2 \\
.3 \\
.4 \\
.5 \\
.6 \\
.7 \\
.8 \\
.8\end{array}$ & $\begin{array}{l}\mathbf{5 4} \cdot 98 \\
\mathbf{5 4} \cdot 89 \\
\mathbf{5 5} \cdot 02 \\
\mathbf{5 5} \cdot 16 \\
\mathbf{5 5} \cdot \mathbf{2 5} \\
\mathbf{5 5 \cdot 3 0} \\
\mathbf{5 5 \cdot 3 4} \\
\mathbf{5 5 \cdot 3 4} \\
\mathbf{5 5 \cdot 3 0} \\
\mathbf{5 5} \cdot \mathbf{2 3}\end{array}$ & $\begin{array}{l}-.11 \\
-.19 \\
-.08 \\
-.01 \\
+.01 \\
+.02 \\
+.04 \\
+.06 \\
+.11 \\
+.11\end{array}$ & $\begin{array}{c}\mathbf{5 5} \cdot 13 \\
\mathbf{5 4} \cdot \mathbf{9 5} \\
\mathbf{5 4} \cdot 89 \\
\mathbf{5 5} \cdot 05 \\
\mathbf{5 5} \cdot \mathbf{2 3} \\
\mathbf{5 5} \cdot \mathbf{3 1} \\
\mathbf{5 5 \cdot 3 5} \\
\mathbf{5 5 \cdot 3 6} \\
\mathbf{5 5 \cdot 3 4} \\
\mathbf{5 5 \cdot 2 9}\end{array}$ & $\begin{array}{r}+.06 \\
-.08 \\
-.17 \\
-.06 \\
+.05 \\
+.04 \\
.00 \\
+.04 \\
+.08 \\
+.11\end{array}$ & $\begin{array}{l}\mathbf{5 5} \cdot 21 \\
\mathbf{5 5} \cdot 12 \\
54 \cdot 99 \\
54 \cdot 93 \\
55 \cdot 10 \\
55 \cdot 29 \\
55 \cdot 38 \\
55 \cdot 40 \\
55 \cdot 38 \\
\mathbf{5 5 \cdot 3 1}\end{array}$ & $\begin{array}{l}+.14 \\
+\cdot 10 \\
-.03 \\
-.14 \\
-.06 \\
+.01 \\
+.01 \\
+.04 \\
+.09 \\
+.11\end{array}$ & $\begin{array}{l}\mathbf{5 5} \cdot 20 \\
\mathbf{5 5} \cdot 07 \\
\mathbf{5 4} \cdot 96 \\
54 \cdot 88\end{array}$ & $\begin{array}{l}+.09 \\
+.06 \\
-.08 \\
-.16\end{array}$ \\
\hline
\end{tabular}

This result was kindly reported by Prof. Bianchi, the Director. This series of latitude observations was made at the observatory of Merate with the Bamberg transit instrument $(55 / 570)$. The residuals subtracted by the computed ones based upon the international latitude service are local $z$ which is given under the heading $\mathrm{O}-\mathrm{C}$ in the above table.

In the report the director adds the following words:

"The residuals are in good agreement with the ones obtained in I9I2-13 at Rome and I9I2-I7 at Greenwich. The causes that determine their systematic character seem to be rather complex. However, a comparison with the temperature variations makes it probable that the thermal gradient near the instrument is the main cause of the observed residuals. This is shown in the observations of $\alpha$ Aurigae, including those made in the day-time."

\section{Preliminary Results of Observations made with Pulkovo Zenith Telescope during the Four Years I929-32.}

The following values of the variation of latitude and $z$ at Pulkovo were taken 
directly from those given in Tables 3 and 4 on p. 6, Poulkovo Observatory Circular, No. I2.

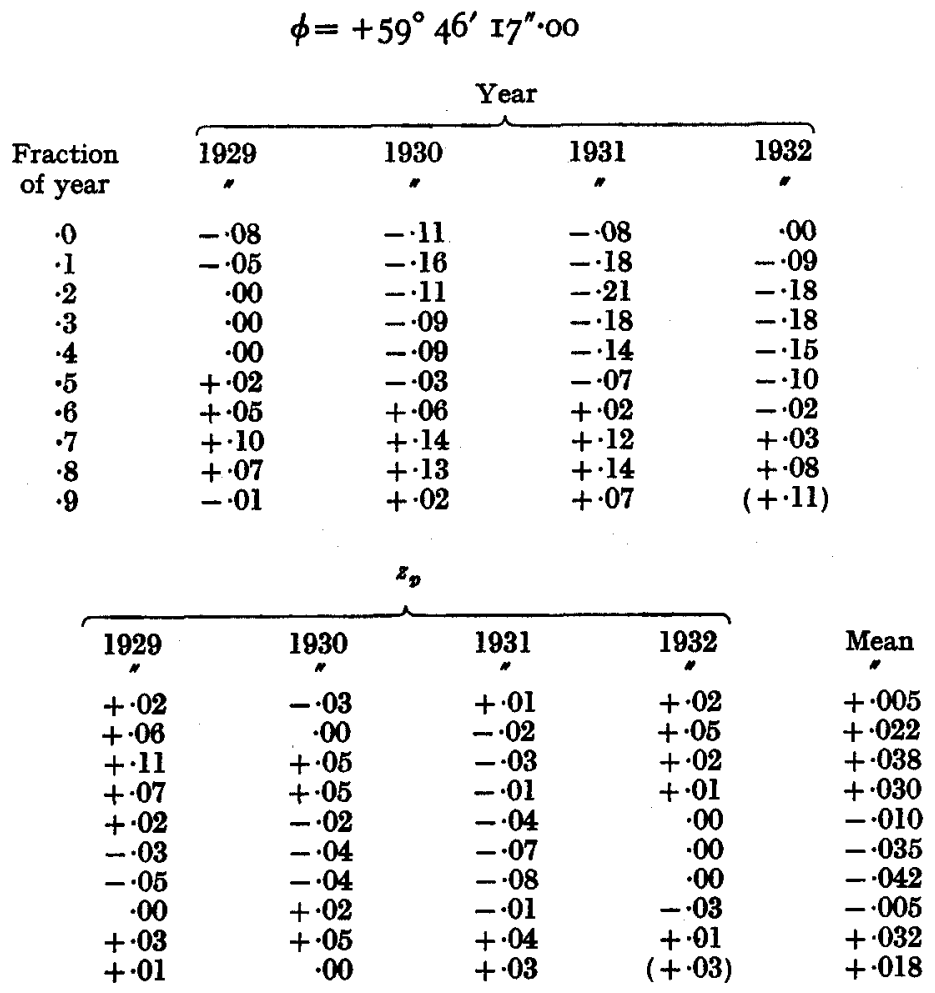

The star programme consists of twelve groups, each of them covering about 2 hours in Right Ascension and containing from seven to ten pairs. The observing programme is similar to that of the International Latitude Service in summer; but in winter the time of observations has been shifted toward the evening, because sometimes more than two groups were observed simultaneously when the weather conditions allowed. It is noteworthy that the general run of the mean $z$ is very similar to those in most stations in the northern hemisphere.

ro. Variation of Latitude at Dehra Dun (India).

$$
\phi=+30^{\circ} \mathrm{Ig}^{\prime}, \lambda=-78^{\circ} 3^{\prime}
$$

This series of observations was made by the Geodetic Branch, Survey of India, in the years I930-33. The work has now been discontinued. The star programme consists of six groups, each of which extends over about 2 hours of R.A., the intervals between two consecutive groups lying from $I^{\mathrm{h}}$ to $3^{\mathrm{h}}$. The detailed reports were given in the Anmual Geodetic Report. The results given below for each tenth of a year were read from the smoothed curve on the plotted observed points drawn by the Geodetic Branch. The plate giving these observing points was 
kindly sent to the Central Bureau by Colonel R. H. Philimore, Director, Geodetic Branch.

\begin{tabular}{ccccc}
\multicolumn{5}{c}{$\phi=+30^{\circ} I 8^{\prime} 5 I^{\prime \prime} \cdot 00$} \\
$\begin{array}{c}\text { Fraction } \\
\text { of year }\end{array}$ & 1930 & 1931 & 1932 & 1933 \\
.0 & - &. &. & Year \\
.1 & - & .71 & 1.06 & 1.11 \\
.2 & .46 & .56 & 1.00 & 1.06 \\
.3 & .34 & .41 & .86 & .94 \\
.4 & .30 & .26 & .66 & \\
.5 & .28 & .19 & .50 & \\
.6 & .30 & .19 & .44 & \\
.7 & .42 & .30 & .55 & \\
.8 & .74 & .53 & .82 & \\
.0 & .78 & 1.07 & .00 & \\
.9 & .78 & 1.10 &
\end{tabular}

Taking the mean latitude as $5 \mathrm{I}^{\prime \prime} \cdot 69$, and subtracting the variation of latitude computed by $X$ and $Y$ of the International Latitude Service from the above tabulated values we got the following local $z$ :

\begin{tabular}{|c|c|c|c|c|c|}
\hline \multirow[b]{2}{*}{$\begin{array}{l}\text { Fraction } \\
\text { of year }\end{array}$} & \multicolumn{4}{|c|}{$z_{d}$} & \multirow[b]{2}{*}{ Mean } \\
\hline & 1930 & 1931 & ${ }^{1932}$ & 1933 & \\
\hline $\begin{array}{l}.0 \\
.1 \\
.2 \\
.3 \\
.4 \\
.5 \\
.6 \\
.7 \\
.8 \\
.9\end{array}$ & $\begin{array}{l}- \\
-.08 \\
-.18 \\
-.23 \\
-.31 \\
-.37 \\
-.33 \\
-.04 \\
+.01\end{array}$ & $\begin{array}{l}+.01 \\
-.07 \\
-.16 \\
-.26 \\
-.31 \\
-.34 \\
-.31 \\
-.18 \\
+\cdot 18 \\
+.26\end{array}$ & $\begin{array}{l}+\cdot 26 \\
+.27 \\
+\cdot 24 \\
+\cdot 12 \\
.00 \\
-.06 \\
+\cdot 02 \\
+\cdot 23 \\
+\cdot 34 \\
+\cdot 40\end{array}$ & $\begin{array}{l}+\cdot 38 \\
+\cdot 32 \\
+\cdot 27 \\
= \\
= \\
= \\
=\end{array}$ & $\begin{array}{l}+.217 \\
+.173 \\
+.068 \\
-.107 \\
-.80 \\
-.237 \\
-.220 \\
-.093 \\
+.160 \\
+.223\end{array}$ \\
\hline
\end{tabular}

It is interesting that the general run of $z$ is very similar to that of Washington or Pulkovo given before. Only the peculiarity in this station is the largeness of the amplitude. This may be caused by, not only the abnormal variations of refraction, but also the real change of the plumb-line due to the special topographical condition to be expected under the Himalayan influence. Moreover, it is noted that an abrupt change of the mean latitude has happened in an epoch about I93r.8.

II. Besides the above-mentioned stations, there is still another one, namely the observatory at Rio de Janeiro, in which the observations of the variation of latitude have been carried out for many years.

\section{B. Recommendations from Members of the Commission}

(a) From Dr R. Schumann, Bemerkungen über Berechnung und Beobachtung der Schreankungen von Stationspolhöhen nebst Vorschlägen

I. Bekanntlich zeigen sich von Anfang an in dem Beobachtungsmaterial zur Breitenvariation, reduciert nach dem seit 40 Jahren bei der Internationalen Erdmessung üblichen Verfahren, ausser der I2- und der 14-monatigen Periodicität 
noch andere "Fluktuationen." Die dafür verwendeten Ausdrücke: systematische Abweichungen, Missstimmigkeiten u.s.w. besagen zu wenig; nach meiner Meinung sind als äquivalente oder koordinierte Naturerscheinungen zu werten:

(I) die Schwankung der Stationspolhöhen, die üblicherweise in eine r2- und eine I4-monatige sinusartige Schwankung aufgelöst wird;

(2) die ständigen, stetigen Schwankungen in den Schlussfehlern;

(3) die ständigen, stetigen $\mathrm{Ab}$-, seltener Anstiege in den Grössen $\Sigma \Delta \Phi$ nebst ihren übergelagerten, eigenen Schwankungen.

Die unter (2), namentlich aber die unter (3) genannten Erscheinungen verdienen, ebenso ausführlich zur Kenntnis der Geophysiker und Astronomen gebracht zu werden, wie die unter (I) genannten, und dies umsomehr, als sie sich in Strenge frei von den Deklinationsverbesserungen $\Delta \delta$ berechnen lassen. Es ist seit langem bekannt und aus den Bänden I bis vi der Ergebnisse des Internationalen Breitendienstes ersichtlich, welche Schwierigkeiten bei der Berechnung dieser an und für sich nebensächlichen Grössen $\Delta \delta$ von Anfang an entstanden sind.

II. Ein ständiges Ansteigen einer $\Sigma \Delta \Phi$ Kurve ergab sich bereits Igor aus der Schnauder-Hecker'schen Polhöhenreihe in Potsdam für die Jahre I894-rgoo, danach fortlaufend bei sämtlichen Stationen des internationalen Parallels*, aber auch die zehnjährige, im I. Vertikal beobachtete Hill'sche Reihe zeigte $\dagger$ einen ständigen Anstieg unter Schwankungen. Im Jahre I93I fand Herr Ingenieur Gsöllpointner dieselbe Eigenschaft aus den Breitenbeobachtungen von Weltevreden; ; die Grössen $\Sigma \Delta \Phi$ werden von ihm mit $[\Delta \phi]$ bezeichnet.

Der wesentliche Vorzug jeder dieser Polhöhen-Aenderungen $\Delta \Phi$ (oder $[\Delta \phi]$ nach Gsöllpointner) liegt darin, dass sie, als Differenz zwischen den beiden Mitteln aus den beiden Abschnitten einer und derselben Gruppe, in Strenge frei ist von den $\Delta \delta$. Die Zwischenzeit zwischen den mittleren Epochen jener beiden Abschnitte ist durchschnittlich 4 Wochen; bei den Schlussfehlern dagegen wird Unabhängigkeit von den $\Delta \delta$ erst erreicht nach Summation von je I2 Unterschieden der Mittel zweier benachbarten Gruppen eines Tages.

In den Astronomischen Nachrichten, Nr. 5813, S. 67 u.f. setzt Herr Dr Ledersteger eine $\Delta \delta$-freie Methode zur Ableitung einer "Polbahn" auseinander, wobei eine bei dieser Summation unvermeidliche Anfangskonstante ausser Betracht bleiben darf. Das Lederstegersche Verfahren habe ich in Nr. 5960, S. I25 analytisch begründet und sodann die Lederstegersche Abstiegsfunktion $J$ in Nr. 6022, S. 339 durch eine zweite Methode kontrolliert, siehe Figur. Diese Methode erlaubte nebenbei jene Anfangskonstante zu bestimmen, und führte zu einer Bestimmung der Polhöhenschwankung einer einzelnen Station, frei von den $\Delta \delta$. Die Vergleiche zwischen den 3 auf verschiedenen Wegen gefundenen Schwankungsreihen der Polhöhenstationen des internationalen Parallels findet man in Nr. 6023, S. 359382, weitere numerische Untersuchungen darüber in Nr. 6027. Die Ableitung einer "Polbahn" habe ich unterlassen, da mir die dazu erforderliche Voraussetzung einer "Rotationsachse eines starren Erdkörpers" nicht gesichert zu sein scheint.

III. Als Ursache der Polhöhenschwankungen wird zwar allgemein eine kleine Bewegung einer Rotationsachse der Erde angenommen; es ist aber zweifelhaft, ob

* Ergänzungsheft Nr. 11 der A.N. Kiel, 1906. Eine numerische Untersuchung über die den zukommende Genauigkeit wird demnächst an anderer Stelle erscheinen.

+ A.N. 229.

$\ddagger$ Jaurverslag von den Topografischen Dienst in Nederlandsch-Indië, 1932, siehe auch A.N. Nr. 6023, S. 383, Zusatz. 
für die in sich bewegliche Erde eine Rotationsachse besteht, und ob die Erde so starr ist, dass die Beobachtungen von Polhöhenschwankungen auf dem 39. Parallel um viele tausende von Kilometern, bis zu den Polen, übertragen werden dürfen. Nach meiner Ansicht bleiben diese Messungen an die jeweilige BeobachtungsStation gebunden.

Sowohl die Ursache der Schlussfehler steht noch nicht fest, als wie jene für die Ab- oder Anstiege der $\boldsymbol{\Sigma} \Delta \boldsymbol{\Phi}[\Delta \phi]$; jedenfalls aber ist bemerkenswert, dass diese bei den verschiedenen Erscheinungen in verschiedener Weise mit den Tagesepochen der Gruppenmittel zusammenhängen. Vielleicht genügt eine Ursache.

Deshalb ist es von grossen Wert, dass die Beobachtungen zur Polhöhenschreankung über die Nacht ausgedehnt werden und in allen Jahreszeiten zu gleichen, aequidistanten Tageszeiten stattfinden.

IV. Die in den Bänden I bis vi der "Ergebnisse..." wiedergegebene Reduktion der Beobachtungen auf dem 39. Parallel blieb bisher die gleiche im allgemeinen; allerdings wurden, wegen neuerlich hervortretender "Missstimmigkeiten in Bd. vI, S. 218 u.f. die Grössen $\Delta_{N}$ eingeführt" : "Es wurden also keine Deklinationskorrektionen durch Mittelbilden über den ganzen Zeitraum abgeleitet, sondern es wurde von Fall zu Fall mit einer individuellen, mit der Zeit auch für die einzelnen Gruppen $N$ veränderlichen Korrektion $\Delta_{N}$ gearbeitet."

Die Tabelle der $\Delta_{N}$ für I9I2 bis I922 (S. 2I9) lässt erkennen, dass sie sowohl mit den Jahreszeiten, als auch mit den Jahren veränderlich sind. Eine Folge dieser Abweichung vom früheren Verfahren ist eine Verzerrung* der Periodicität in den Schwankungen der Stationspolhöhen, beginnend mit dem Zeitpunkte der Einführung, hier I9I2. Dies wird ersichtlich bei einem Vergleich zwischen den Schwankungskurven aus den internationalen Polhöhenmessungen mit denen nach den beiden oben unter II genannten $\Delta \delta$-freien Methoden. Den Verlauf ihrer Unterschiede zeigt beifolgende Figur; der Einfachheit wegen wurden die Epochen gleichabständig genommen, I mm. entspricht I/I2 Jahr.

Die bevorzugte Station Carloforte eignet sich am besten zu solchen Untersuchungen, da bei ihr infolge der grösseren Anzahl von Sternpaaren und Abenden der Einfluss der reinen Beobachtungs-Unsicherheit zurücktritt.

Die unterste Kurve zeigt den Verlauf der Differenzen der Polhöhenschwankungen zwischen den beiden $\Delta \delta$-freien Methoden und zwar im Sinne: zweite minus dritte Methode, mit der Bezeichnung nach A.N. Nr. 6022, S. 337 u.f. also:

$$
S_{14}-L r=\left(\Sigma \Delta \Phi-\frac{1}{14} S\right)-(\Sigma \Delta \Phi-J)=J-\frac{1}{14} S
$$

da beide Methoden auf den $\Delta \delta$-freien $\Sigma \Delta \Phi$ beruhen, gibt mithin die unterste Kurve zugleich die Unterschiede zwischen den beiden, ebenfalls $\Delta \delta$-freien, von einander unabhängig berechneten Abstiegsfunktionen $J$ und $\frac{1}{14} S$; die hier nicht interessierenden, oben erwähnten Summations-Konstanten sind dabei berücksichtigt. Der ruhige Verlauf dieser Kurve rührt übrigens davon her, dass bei $\frac{1}{14} S$ die Gruppenmittel-Differenzen über I4 Monate, bei den $J$ solche über I2 Monate successive summiert und gemittelt werden. Wesentlich ist, dass die Aenderungen im Deklinations-System zwischen rgoo und 1922 ohne Einfluss bleiben.

Die oberste Kurve stellt dar die Schwankungs-Differenzen im Sinne: erste minus zweite Methode, oder: $\phi_{\text {1nt }}-\left(\Sigma \Delta \Phi-\frac{1}{14} S\right)$, die mittlere Kurve: erste minus dritte Methode, oder: $\phi_{\text {int }}-(\Sigma \Delta \Phi-J)$; beide Kurven werden unmittelbar durch Aenderungen in der Behandlung der Deklinationen und ihrer Verbesserungen betroffen,

* Siehe A.N. Nr. 5961, Tab. 10, 11, Fig. 2, Tab. 13. 


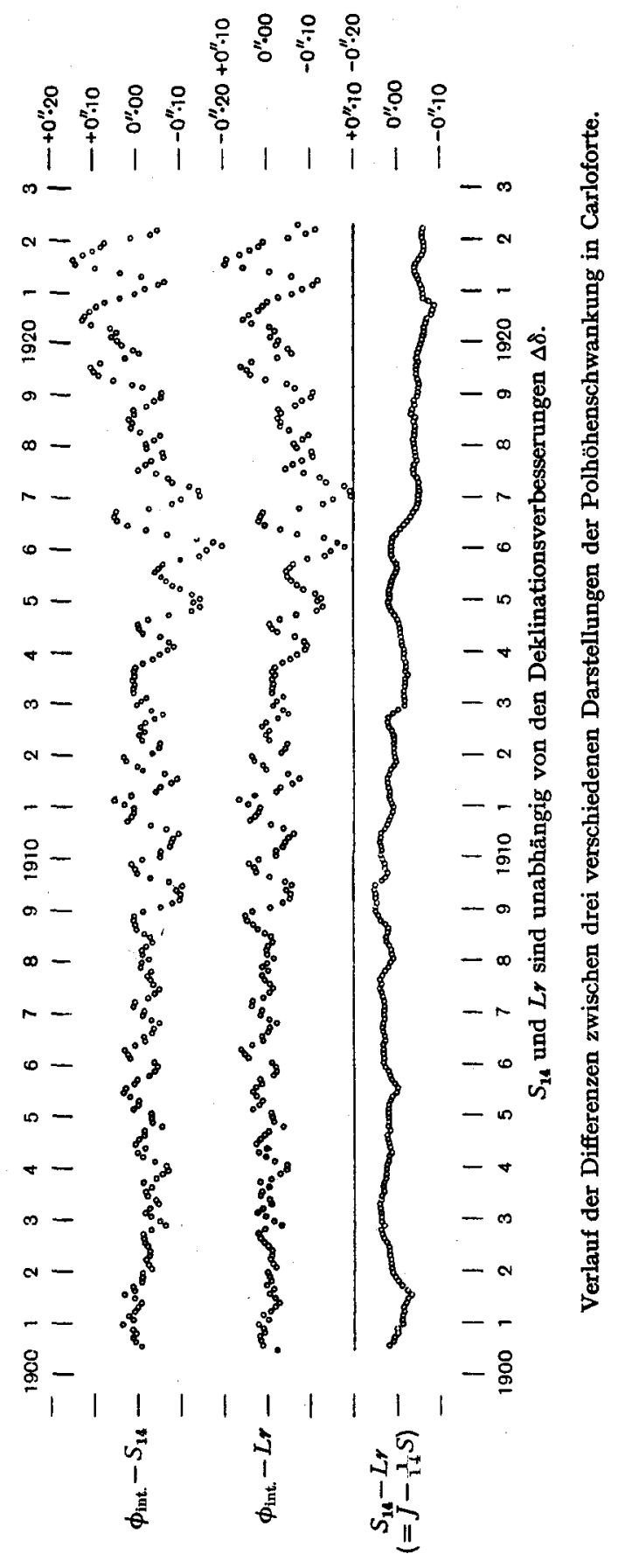

I2I 
deren Bestimmung bekanntlich von Anfang an in das übliche Reductions-Verfahren mit eingeschlossen worden ist.

Aenderungen im Deklinations-System fallen bekanntlich wenig ins Gewicht, wenn man es vorzieht, aus Messungen auf dem 39. Parallel eine Bahn für den fast $6000 \mathrm{~km}$. entfernten Nordpol zu berechnen. Im Interesse der Schwankungen der auf dem 39. Parallel unmittelbar beobachteten Stations-Polhohen dagegen entsteht der Wunsch: die Beobachtungen möchten von $\mathrm{I} 900$ an wenigstens nach gleichen Ver fahren bezüglich der Deklinationen durchgerechnet werden.

V. Im Laufe der letzten Jahre wurde von verschiedenen Seiten auf Veränder lichkeiten in geographischen Längen bei Gelegenheit schärfsterneuerer Zeitvergleiche hingewiesen; die Beträge der Aenderungen sind von der gleichen Grössenordnung, wie die der in nordsüdlichen Richtungen erfolgenden Polhöhenschwankungen. Wichtiger als ein selbstständiges Stations-Netz für die Ostwest-Komponenten allein erscheint es, die internationalen Stationen für Polhöhenschroankung zugleich in Länge fortlaufend auf das schärfste zu vergleichen. Dann würde wenigstens für diese Stationen die Beweglichkeit in den beiden Hauptrichtungen erkannt werden; jedenfalls war der Vorschlag sehr zu begrüssen, diese Stationen auch mit Instrumenten für seismische Beobachtungen auszustatten.

Wenn die Konstanz der Rotationsdauer der Erde geprüft werden soll, so ist es wichtig, die Beweglichkeit oder Unbeweglichkeit der dazu ausersehenen Beobachtungsstationen $\mathrm{zu}$ verfolgen.

Wien, 1934 Oktober

(b) From Dr H. S. Jones, Astronomer Royal

Latitude Variation observations at Greenwich with the Cookson Floating Telescope have continued regularly on the same lines as previously. I propose to bring this programme to a termination about the end of I935, after 25 years' observations. The telescope will then be rehoused, on a site about 300 yards from the present site and adjacent to the new transit circle.

There is a marked dependance of the latitude variation observed at Greenwich upon the wind direction. In its new position, the telescope will not be so near the brow of a steep hill and the ground will fall away to the north more gradually. It will be of interest to find out whether the $z$-term is appreciably changed by the change in location of the instrument.

At the same time, the present programme, designed to determine the aberration constant as well as the variation of latitude, will be modified.

The new programme will be more similar to the present International programme; three groups of stars, centred near midnight, will be observed nightly. A full discussion of the results of the present programme will be made in due course.

\section{(c) From Prof. E. Esclangon, Director of the Paris Observatory}

I. Il est important d'avoir les résultats détaillés du Service International des Latitudes depuis I922, le plus tôt possible, ainsi que les coordonnées du pôle instantané dès le début du Service International des Latitudes réduites au même pôle moyen. 
2. Il est important que les coordonnées provisoires du pôle soient déterminées en tenant compte non seulement des stations du nord, mais aussi de celles du sud.

3. Il est désirable d'avoir la troisième station dans l'hémisphère sud (en Afrique du Sud), en plus d'Adélaide et de La Plata. Mesures pour que les stations existantes travaillent régulièrement.

\section{(d) From Dr H. KIMURA}

To obtain $z$ in any isolated station, the value of one division of the micrometer is of the first importance. Nevertheless, its accurate determination is the most difficult one. As is well known, there are two methods for its determination, namely, one by the observations of the greatest elongations of circumpolar stars and the other by the measurements of the declination-differences of two stars having nearly the same right ascensions. Of these two methods the latter one only may be used universally in any place in the world, and the necessary and important thing for this method is the exactness of the angular value of the declinationdifference. It is, however, generally exceedingly uncertain except for some which were specially determined for the purpose. But those special scale-stars are mostly of high northern declinations, so they can hardly be used in southern stations.

The present distribution of the international latitude stations over the world is within the limit of latitude $+39^{\circ}$ to $-35^{\circ}$, and the best manner of finding relative values of the micrometers for all these stations is to observe the same scale-stars at these places. Moreover, such stars must be chosen in the equatorial zones. Now as the constants referred to the micrometers at all stations in the north parallel may be well determined, the angular value of the declination-difference for any scale-stars will also be well found from the observations made on them. Thence the values of one division of the micrometer for other stations on the equator and in the southern hemisphere may be determined. And at the same time, such universal co-operation might be of some service for the examination of the results of the greatest elongations in both hemispheres.

On my request, Mr Goto, observer in Midusawa Observatory, has chosen plenty of such scale-stars after having examined for double and variable stars. Their catalogue is annexed to this report.

I hope all observers who are engaged on the latitude observations in the international stations will agree on this plan and will put it into practice.

\section{(e) From Dr W. BowIE, Head of the Geodetic Section of the Coast and Geodetic Survey, U.S.A.}

Of general recommendations in compliance with your request of September 22, I934, I have only one and it is a suggestion rather than a recommendation. You need not be told of the current tendency to have photographic observations replace visual ones. This is likely to affect the programme of the International Latitude Service and I suggest that the Committee consider the subject of photographic observations.

One of the primary reasons for the organization of the International Latitude Service was homogeneity and uniformity, not only of stars used but also in instruments, observatories and methods. Under financial conditions even less unfavourable than those of the present it would probably be impracticable to procure an 
adequate supply of photographic instruments of the same type and to place them in operation simultaneously at all observatories. This would mean that any change from visual to photographic methods would have to be made gradually, the photographic observations being first added to the programme, perhaps one observatory at a time until all observatories were equipped with photographic apparatus and it would be possible to abandon visual work entirely, if that seemed desirable.

The Talcott visual method of observation has remained unchanged in substance through many decades, but there is no guarantee that the approved type of photographic observation will be equally stable. The gradual installation of photographic equipment with a view to the final displacement of visual observations might involve a choice between:

(a) a possibly outmoded type of photographic apparatus at all observatories of the International Latitude Service;

(b) a lack of homogeneity in the photographic apparatus used.

I have no suggestions as to the type of photographic apparatus to recommend at this time and merely lay the whole problem before the members of the Committee, to whom doubtless similar ideas have occurred.

\section{(f) On the Instrumental Adjustment of a Zenith Telescope. By EARL L. WILLIAMS}

In the instruments used in the International Variation of Latitude Observatories the telescope is fixed at the end of a long horizontal axis supported at two points near its centre. This form of mounting, with the heavy telescope on one end and a counterweight on the other, gives rise to bending of the axis, with an attendant extra instrumental constant known as "side flexure."

Without offsetting adjustments, which adjustments are the subject of this paper, the effect of this flexure will be to make the middle thread of the telescope fail to coincide with the meridian at the zenith, but it will do so at the horizon. The customary method* of offsetting this effect has been to adjust the collimation to the negative of the flexure, thus bringing the middle thread to the meridian at the zenith, but causing an increasing departure from the meridian as zenith distance increases. This arises from the fact that the coefficients of flexure and collimation are numerically alike in the zenith but nowhere else. Since great accuracy in this particular is not required for the variation of latitude programme, where all the stars used transit within $25^{\circ}$ of the zenith, this lack of conformity to the meridian at all zenith distances is not important, but there is an appreciable error when the telescope is close to the horizon as when sighting on the meridian mark. The error in the meridian mark resulting may be of importance, as when it is being used as an azimuth mark, as has recently been the case at Gaithersburg.

Proposed below is a more perfect method of offsetting the effect of flexure, which avoids completely any disadvantages of the method just reviewed, since in this the middle thread can be made to follow the meridian perfectly at all zenith distances.

In addition, it is possible to save considerable time in keeping the instrument in sufficiently good adjustment since computation of instrumental constants is unnecessary.

In the method instituted at Gaithersburg, instead of having the horizontal axis level and at right angles with the vertical axis, the horizontal axis is raised at the

* Method discussed by Ross, Astr. Nach., 4538, Nov. I9II. 
telescope end by an amount equal to the flexure, the vertical axis remaining undisturbed. Because the coefficient of the inclination (of the horizontal axis) is identically the same coefficient as that for flexure, perfect coincidence with the meridian is obtained at all zenith-distances, the meridian mark included. Other constants, such as collimation, are now made zero.

\section{Activities of the Central Bureau at Mizusawa}

The present work in the Central Bureau is just the same as reported in Transactions of the I.A.U. 4, II4, I932, except for the last one (6).

The evaluations of each individual value of latitude observed at the three old stations, namely, Midusawa, Carloforte and Ukiah during about 8 years I922.7I93I.0, and the definitive values of the co-ordinates of the polar curve and local $z$ for each station; in addition, the determination of the errors for the adopted values of the declinations and the proper motions of all pairs from the latitude observations themselves were entirely finished, and their results are now in the press.

\section{Report on the Financial Position}

During the past three years, the Central Bureau of the International Latitude Service at Mizusawa has received the following grants from the International Astronomical Union and the Geodetic Section of the International Geodetic and Geophysical Union:

\begin{tabular}{|c|c|c|c|}
\hline \multicolumn{2}{|c|}{ From I.A.U. } & \multicolumn{2}{|c|}{$\begin{array}{c}\text { From } \\
\text { Geodetic Section }\end{array}$} \\
\hline $\begin{array}{l}\text { Swiss } \\
\text { francs }\end{array}$ & $\begin{array}{c}\text { For the } \\
\text { year }\end{array}$ & Francs & $\begin{array}{l}\text { For the } \\
\text { year }\end{array}$ \\
\hline $\begin{array}{l}\mathbf{2 , 5 0 0} \\
\mathbf{2 , 5 0 0} \\
\mathbf{2 , 5 0 0} \\
\end{array}$ & $\begin{array}{l}1933 \\
1934 \\
1935\end{array}$ & $\begin{array}{l}7,000 \\
7,000 \\
7,000\end{array}$ & $\begin{array}{l}1932 \\
1933 \\
1934\end{array}$ \\
\hline$\overline{7,500}$ & & $\frac{7,000}{28,000}$ & 1935 \\
\hline
\end{tabular}

From Reduced Geodetic Association $\overbrace{\text { Pounds In the }}$ t s. d. year $34173 \quad 1933$

H. KimURA

President of the Commission

March 8, 1935

ERRATA

The two following errata in the recently published " Results of the International Latitude Service from 1922.7 to I93I.0" are to be noted:

Page 20, 1. 2 and 1. 4, for "division" read "revolution". 


\section{CATALOGUE OF SCALE-PAIRS WHOSE DECLINATIONっ ARE VERY NEAR TO ZERO.}

The star-places, precession and secular variation were taken directly from the Albany Catalogue. The proper motions were taken from the Catalogues of Boss and Yale. Under the heading "remarks" the magnitude and distance of the double star are given; these are taken from the Catalogue of Aitken. 


\begin{tabular}{|c|c|c|c|c|c|c|c|c|c|c|c|c|}
\hline 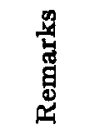 & a 11 & 11 & 11 & 11 & 11 & 11 & 11 & 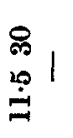 & 11 & 11 & 11 & 11 \\
\hline 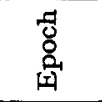 & $\stackrel{9}{\dot{\infty}}$ & 品官 & 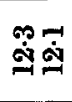 & 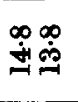 & 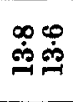 & 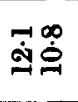 & $\begin{array}{l}\stackrel{\sim}{\infty} \\
\stackrel{\sim}{\sim}\end{array}$ & تُ & $\begin{array}{l}\ddot{0} \\
\dot{m}\end{array}$ & 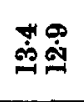 & $\stackrel{\varphi}{\dot{m}} \dot{q}$ & $\begin{array}{l}m \infty \\
\ddot{d} \dot{\infty} \infty\end{array}$ \\
\hline 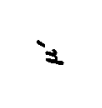 & .11 & 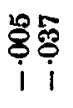 & 11 & 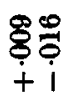 & i & $\overrightarrow{\oint_{i}} \mid$ & 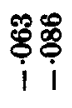 & है & $\begin{array}{l}\text { 응ㅎㅇ } \\
\dot{i}\end{array}$ & 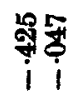 & ఫุ๋ & 趈। \\
\hline 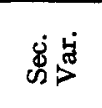 & - & 윻훟 & 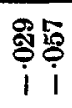 & 苞冬 & \begin{tabular}{l}
$\infty$ \\
\hdashline \\
$i$ \\
$i$
\end{tabular} & 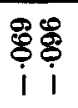 & ํㅜㅇ용 & 호용 & $\begin{array}{l}\infty \\
5 \\
\\
i \\
\end{array}$ & $\underset{i}{\stackrel{9}{9}}$ & $\stackrel{5}{i} \frac{9}{i}$ & $\frac{9}{i} \frac{8}{i}$ \\
\hline 岕 & 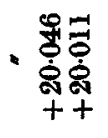 & 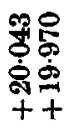 & $\begin{array}{l}\text { क्षे: } \\
\text { क्ष } \\
\text { क़ें } \\
++\end{array}$ & 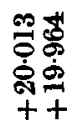 & 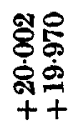 & 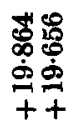 & 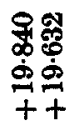 & 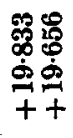 & 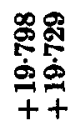 & 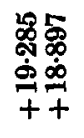 & 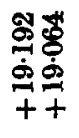 & 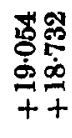 \\
\hline 옹 & 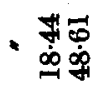 & 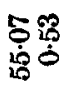 & فำ & 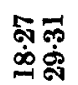 & 웅ํㅇำ & 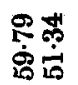 & 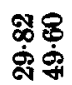 & 声惑它 & 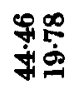 & 䟽 & 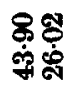 & 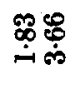 \\
\hline$\frac{i}{8}$ & .05 & ட̊ำ & 퍼여 & 명요 & $\infty$ & 옴 왁 & 路 & 욤 & 8 & $\bar{x} 20$ & $\mathscr{7}$ & 녁 \\
\hline م & - $\stackrel{0}{+\infty}+$ & ฬึָ & $p$ & 77 & จิ & i & $\stackrel{N}{+}+$ & ii & 11 & $\vec{t}+\vec{t}$ & Nิ & พิ \\
\hline$z$ & & 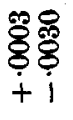 & 11 & 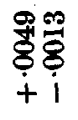 & 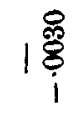 & 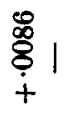 & 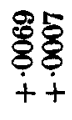 & 兽। & 해용 & 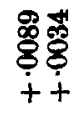 & 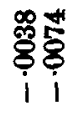 & $\begin{array}{l}\text { 我 } \\
\stackrel{0}{+} \\
+\end{array}$ \\
\hline $\begin{array}{l}\dot{8} \\
\stackrel{5}{5}\end{array}$ & 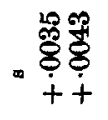 & 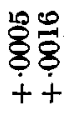 & 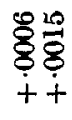 & 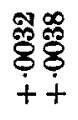 & 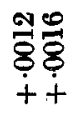 & 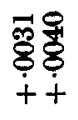 & 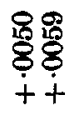 & 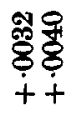 & 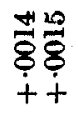 & $\begin{array}{l}\text { 尽萝 } \\
\dot{+}+\dot{+}\end{array}$ & $\begin{array}{l}\text { 켱 웡 } \\
\dot{+}+\dot{+}\end{array}$ & 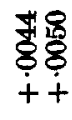 \\
\hline 适 & 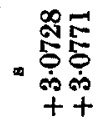 & 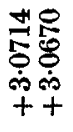 & 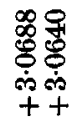 & 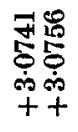 & 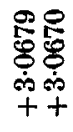 & 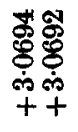 & 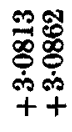 & 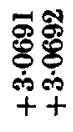 & 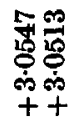 & 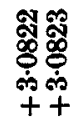 & 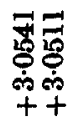 & 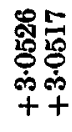 \\
\hline 옹 & 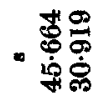 & 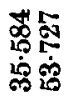 & 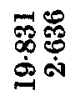 & 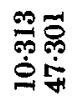 & 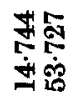 & 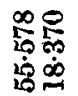 & 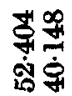 & 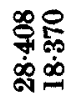 & 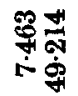 & 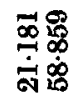 & $\begin{array}{l}\infty \\
\stackrel{\infty}{0} \frac{8}{8} \\
\infty \\
\infty\end{array}$ & 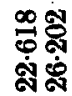 \\
\hline & वOM & m & ㅇำ & ๑요 & 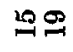 & $8 \%$ & 융 & m & ஜீ & $m 5$ & - 엄 & ษ \\
\hline 14 & $=00$ & 00 & 00 & 00 & 00 & 00 & 00 & 00 & 00 & $\neg-1$ & $\rightarrow m$ & $-\pi$ \\
\hline 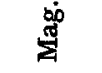 & $\dot{\theta} \vec{x}$ & 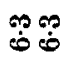 & 움욤 & H'? & $\stackrel{9}{9}$ & $\begin{array}{l}0 \\
\dot{0} \\
\dot{0}\end{array}$ & فُ & $\begin{array}{l}9 \infty \\
\dot{0} \dot{0}\end{array}$ & فㅎㅂ & م & 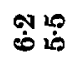 & $\begin{array}{l}\infty \\
0 \\
\dot{0}\end{array}$ \\
\hline 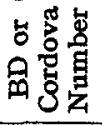 & 实 & 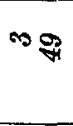 & ริธ & ลัพ & เి & : & $\stackrel{\infty}{\underset{\pi}{\approx}}$ & 25 & 독욤 & สีฬ & ธีำ & 촉격 \\
\hline 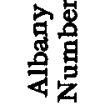 & 옥 & 解 & 氜 & 司芯 & సैं & 韋等 & 我电 & 志题 & 앵옹 & 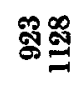 & 동 & 옹영 \\
\hline
\end{tabular}

I28 


\begin{tabular}{|c|c|c|c|c|c|c|c|c|c|c|c|c|}
\hline 莺 & 11 & 11 & 11 & 11 & 11 & 11 & 11 & 11 & 11 & 11 & 11 & 11 \\
\hline 秀 & 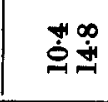 & 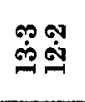 & $\begin{array}{l}\text { क्ष } \\
\text { ஸ் }\end{array}$ & $\begin{array}{l}\infty \\
\dot{\oplus} \\
\dot{\exists}\end{array}$ & 苟灾 & 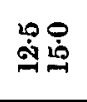 & 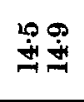 & 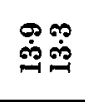 & فำ & 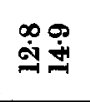 & 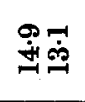 & 它是 \\
\hline 1 & 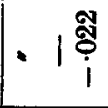 & $\underset{i}{\stackrel{*}{*}}$ । & | $\stackrel{\text { จ }}{+}$ & $\stackrel{\text { F⿻ำ }}{+}$ & 亲 원 & 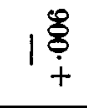 & 1 i & 11 & $\stackrel{\$}{i} \mid$ & $1 \stackrel{8}{\text { : }}$ & 11 & $1 \%$ \\
\hline $\begin{array}{l}\text { 迎 } \\
\text { जे }\end{array}$ & 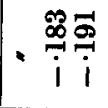 & 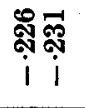 & 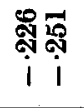 & 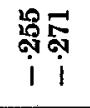 & 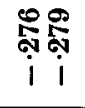 & $\underset{i}{\stackrel{8}{9}} \underset{i}{\stackrel{9}{9}}$ & 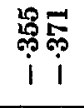 & 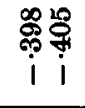 & 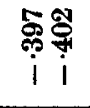 & 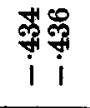 & 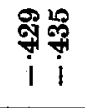 & 果是 \\
\hline 总 & 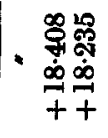 & 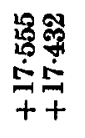 & 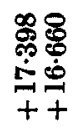 & 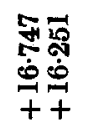 & 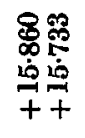 & 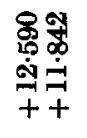 & 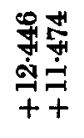 & 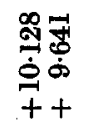 & 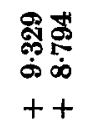 & 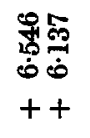 & 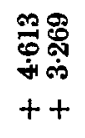 & 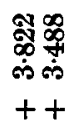 \\
\hline $\begin{array}{l}\text { 옴 } \\
\text { ఫ் } \\
\text { Ф் }\end{array}$ & 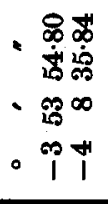 & 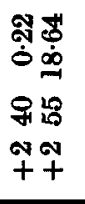 & 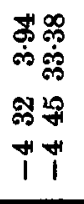 & 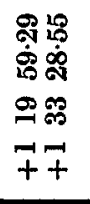 & 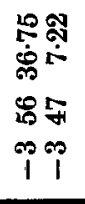 & 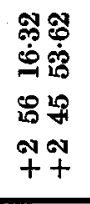 & 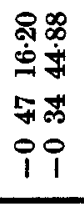 & 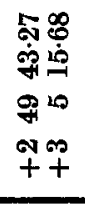 & 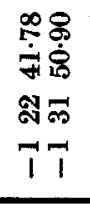 & 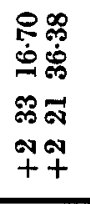 & 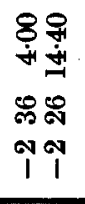 & 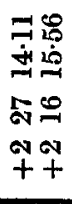 \\
\hline$z$ & 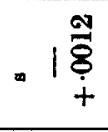 & $\begin{array}{l}20 \\
\stackrel{10}{9} \\
\dot{+}\end{array}$ & 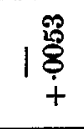 & 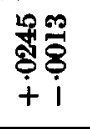 & 훙융 & 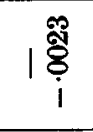 & $1 \stackrel{\mathscr{\dddot { f }}}{\dot{8}}$ & 11 & 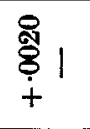 & 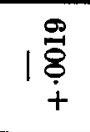 & 11 & 18 \\
\hline 它点 & 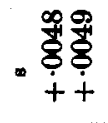 & 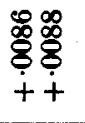 & 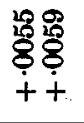 & 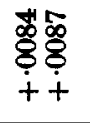 & 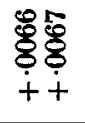 & $\begin{array}{l}\text { 양요 } \\
\text { לุo } \\
\dot{+}+\dot{+}\end{array}$ & 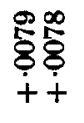 & 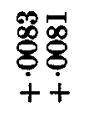 & 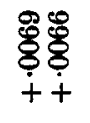 & 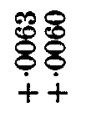 & 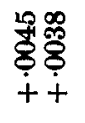 & 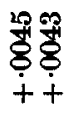 \\
\hline 总 & 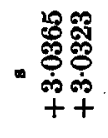 & 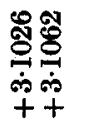 & 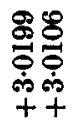 & 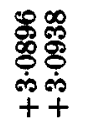 & 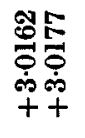 & 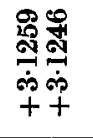 & 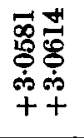 & 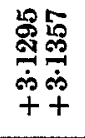 & 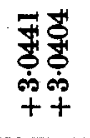 & 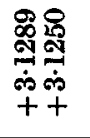 & 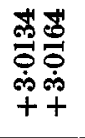 & 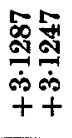 \\
\hline $\begin{array}{l}\stackrel{9}{9} \\
\stackrel{9}{9} \\
\dot{4}\end{array}$ & 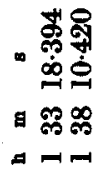 & 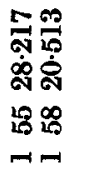 & 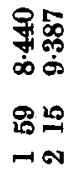 & 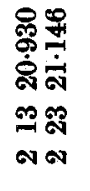 & 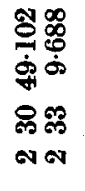 & 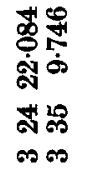 & 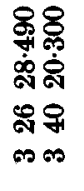 & 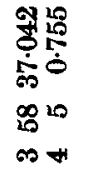 & 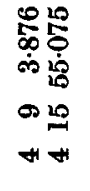 & 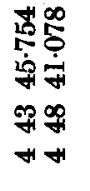 & 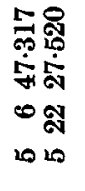 & 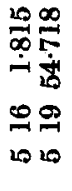 \\
\hline 总 & 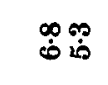 & $\begin{array}{l}\infty \\
\dot{\varphi}\end{array}$ & 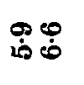 & 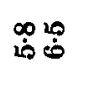 & $\begin{array}{l}\infty \\
0 \\
\dot{\omega}\end{array}$ & $\begin{array}{l}0 \\
0.0\end{array}$ & فُ & فํ. & فे & 5i & فํ & 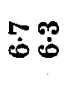 \\
\hline 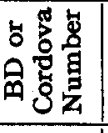 & స్తి త్రి & 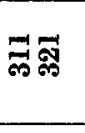 & 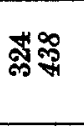 & 악 & 路 & 号喿 & 路总 & 옹 & ్ㅗㅇㅇㅛ & : & 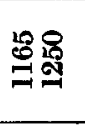 & 芯尔 \\
\hline 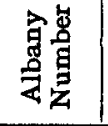 & 实哭 & 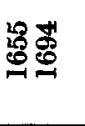 & 串总 & 品巻 & $\begin{array}{l}\text { สั5 } \\
\text { ลิล }\end{array}$ & जo & 总芯 & 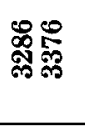 & 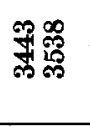 & 需鬲 & 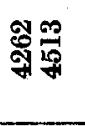 & 㝵桑 \\
\hline
\end{tabular}




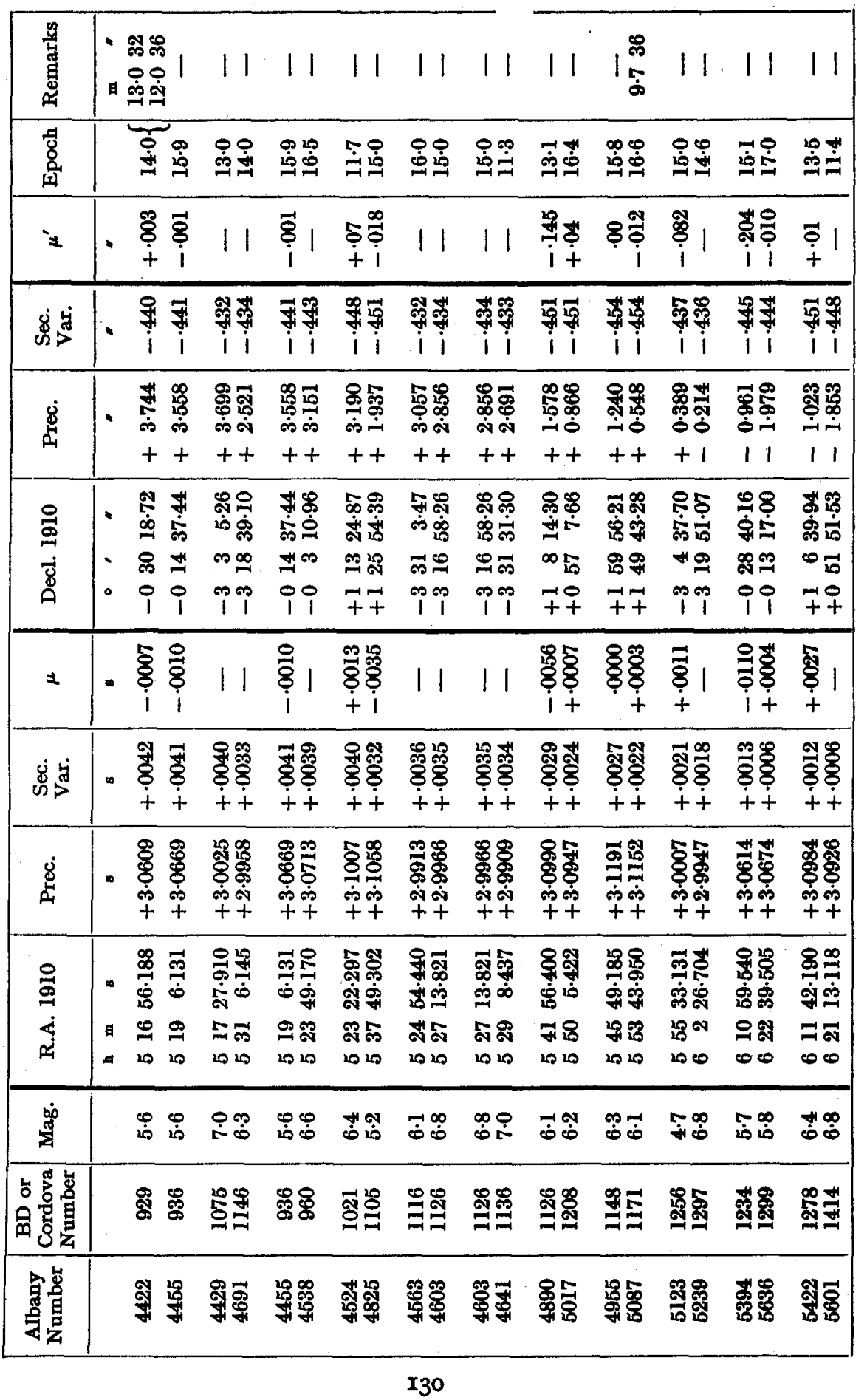




\begin{tabular}{|c|c|c|c|c|c|c|c|c|c|c|c|c|}
\hline 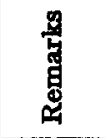 & - 11 & 11 & 11 & 11 & 11 & 11 & 11 & 11 & 11 & 11 & 11 & 11 \\
\hline$\frac{5}{8}$ & ڤึت & 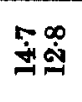 & 官市 & 号灾 & $\overrightarrow{\dot{9}}$ & 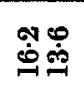 & $\begin{array}{l}\stackrel{9}{9} \\
\dot{\sim}\end{array}$ & $\stackrel{\text { Ṇ }}{\stackrel{\oplus}{\oplus}}$ & مُ & 宄芯 & 囟 & $\stackrel{\sim}{\dot{q}}$ \\
\hline I & .11 & 11 & $\stackrel{ }{8}$ & ஜி & $1 \%$ & ఫ̊̊̊̊ & 11 & 18 & $\stackrel{m}{i} \frac{m}{i}$ & ڤ్̣̂ & $1 \stackrel{8}{i}$ & \& \\
\hline 岕户 & 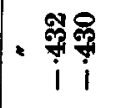 & 羿 & $\begin{array}{l}\text { is } \\
i \\
i\end{array}$ & 岳势 & 葆 & 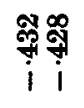 & 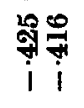 & 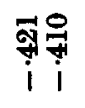 & 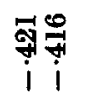 & Đ핳 & 趈题 & 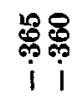 \\
\hline 离 & 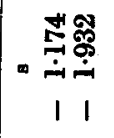 & 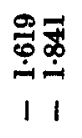 & 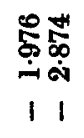 & 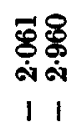 & 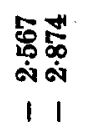 & 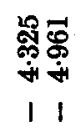 & 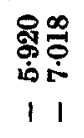 & 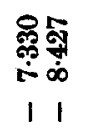 & 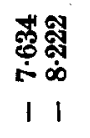 & 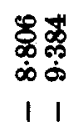 & 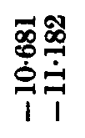 & 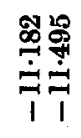 \\
\hline 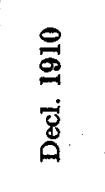 & 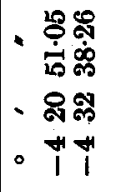 & 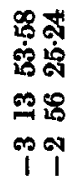 & 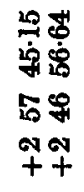 & 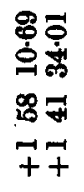 & 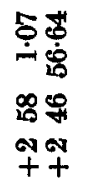 & 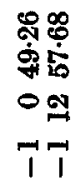 & 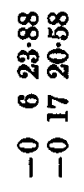 & 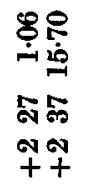 & 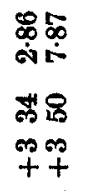 & 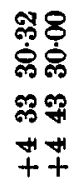 & 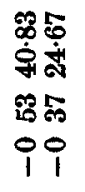 & 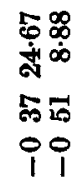 \\
\hline 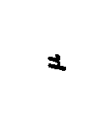 & -11 & 11 & 융ㅇㅀㅇ & 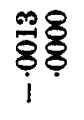 & I 옹 & 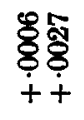 & 11 & 1 ళ్రి & 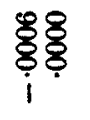 & 乐审 & 1 융 & \&્ઠ \\
\hline \& & 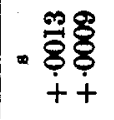 & 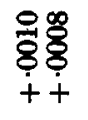 & 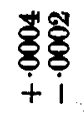 & 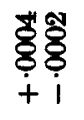 & ర్రికో & 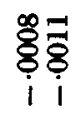 & 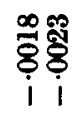 & 密 & 路 & 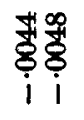 & 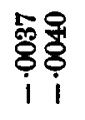 & 웅용 \\
\hline 总 & 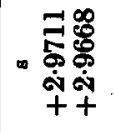 & 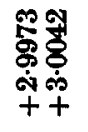 & 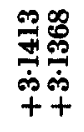 & 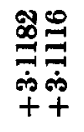 & 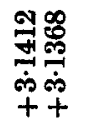 & 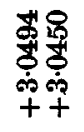 & 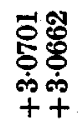 & 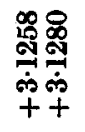 & 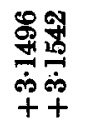 & 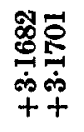 & 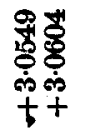 & 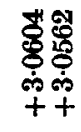 \\
\hline 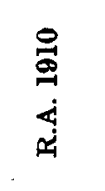 & 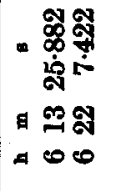 & 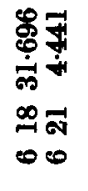 & 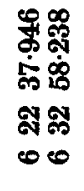 & 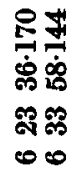 & 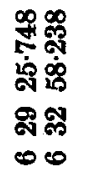 & 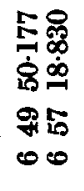 & 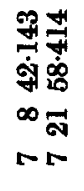 & 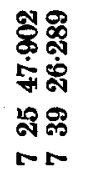 & 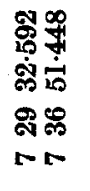 & 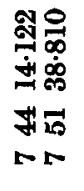 & 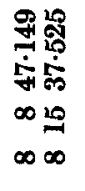 & 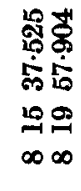 \\
\hline 楽 & فे & ఏ5 & कீ & $\ddot{60}$ & $\begin{array}{l}\infty \\
0 \\
0\end{array}$ & $\underset{\dot{\varphi}}{\mathscr{b}}$ & ro & $\stackrel{9}{\mathscr{1} 0}$ & $\begin{array}{l}\text { कo } \\
\text { is }\end{array}$ & 电 & 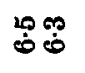 & $\ddot{\theta}$ \\
\hline 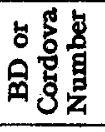 & 역욤 & 承客 & 密恕 & 殸录 & 承点 & 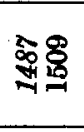 & 옹్ㅀ & 哭志 & 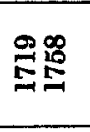 & 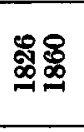 & 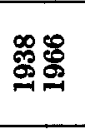 & 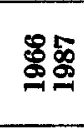 \\
\hline 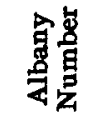 & 肯窇 & 总罟 & 跑 శ్ & 员藏 & 䊀辰 & $\begin{array}{l}208 \\
300\end{array}$ & 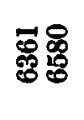 & $\begin{array}{l}\text { 象: : } \\
\text { : }\end{array}$ & 布占 & శ్రీ양 & 式蛋 & 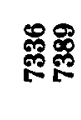 \\
\hline
\end{tabular}




\begin{tabular}{|c|c|c|c|c|c|c|c|c|c|c|c|c|c|}
\hline 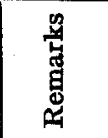 & $a^{\prime} \mid 1$ & 11 & 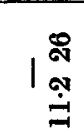 & 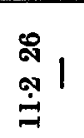 & 11 & 11 & 11 & 11 & 11 & 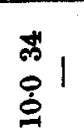 & 11 & 11 & 11 \\
\hline 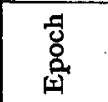 & ஸ્ષ & 宙莤 & 它亩 & 돔 & $\stackrel{9}{\stackrel{9}{9}}$ & 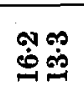 & 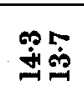 & 崶 & $\stackrel{\varphi}{\dot{\oplus} \mathscr{H}}$ & 电虽 & 冢 & 舅兽 & 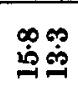 \\
\hline 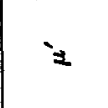 & .11 & 突导 & 11 & 11 & $\begin{array}{l}\text { 궁영 } \\
\dot{+}\end{array}$ & 承 & 11 & $\frac{\text { स̊ํํ }}{i+}$ & જ & 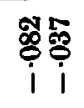 & $\begin{array}{l}\text { ङ્ } \\
\dot{+}\end{array}$ & 쿵ํ & 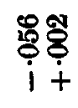 \\
\hline 崖 & - & 笋 & 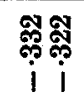 & స్ఞ̊ & 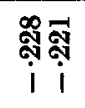 & $\stackrel{\mathscr{\infty}}{\stackrel{\infty}{\mathrm{i}}}$ & $\underset{\mathrm{i}}{\overrightarrow{0}} \overrightarrow{0}$ & i & 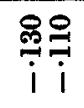 & $\stackrel{\infty}{\underset{i}{i}} \underset{i}{\stackrel{9}{i}}$ & $\begin{array}{l}\infty \\
\stackrel{0}{0} \\
\mathrm{i} \\
\mathrm{i}\end{array}$ & 峉 & क्ष \\
\hline ن⿺辶ّ & 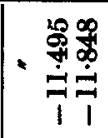 & 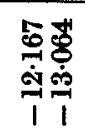 & 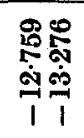 & 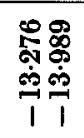 & 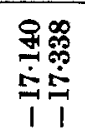 & 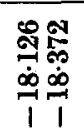 & 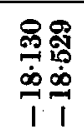 & 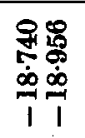 & 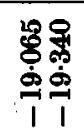 & 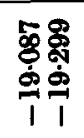 & 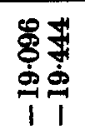 & 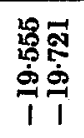 & 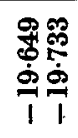 \\
\hline 옹 & $\begin{array}{r}\infty 20 \\
\infty \\
-\infty \\
-\infty \\
\infty\end{array}$ & 实 & 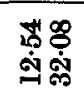 & 号鬲 & ชุํำ & $\stackrel{\infty}{\oplus}$ & $\begin{array}{l}\text { 웅 } \\
\text { ف영 }\end{array}$ & 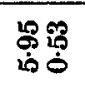 & 耐 & 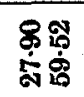 & 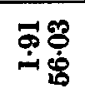 & 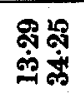 & 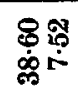 \\
\hline वृं & - & 品荋 & 1007 & 20 & 必 & $\stackrel{\leftrightarrow}{\circ} \varrho$ & 코옹 & $\mathscr{9}$ & $\stackrel{\bullet}{\circ}$ & 욱 & 承苟 & 象㠿 & $\infty$ 落 \\
\hline & - 11 & $\overrightarrow{1}$ & 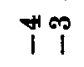 & $i$ & $\dddot{m}$ & ii & $p_{i}$ & †૭ & it & $\overrightarrow{1}$ & $\vec{\imath}$ & 웋 & $\stackrel{N}{+}$ \\
\hline$=$ & & 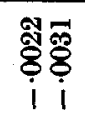 & 11 & 11 & 유용 & 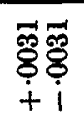 & 11 & 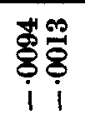 & 氕 & 承 & $\overbrace{i}^{ஜ}$ & 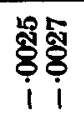 & 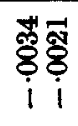 \\
\hline 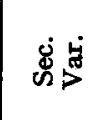 & - 윙ㅎㅇ & 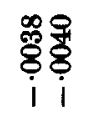 & 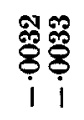 & 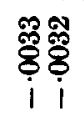 & 處员 & 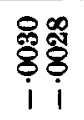 & 흥효 & $\stackrel{\infty}{\stackrel{\infty}{8}}$ & 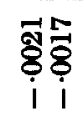 & 율요 & 范 & ఈ̈ & 我声 \\
\hline 总 & 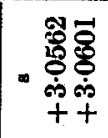 & 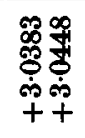 & 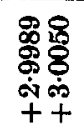 & 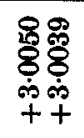 & 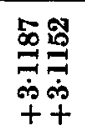 & 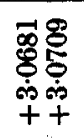 & 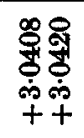 & 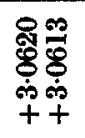 & 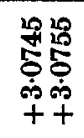 & 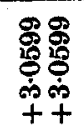 & 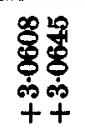 & 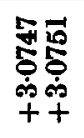 & 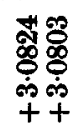 \\
\hline 웅 & 施 & 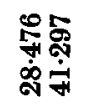 & 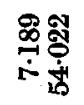 & 实京 & 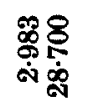 & 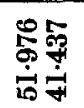 & 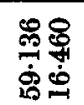 & 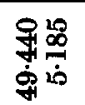 & $\begin{array}{l}80 \\
\frac{8}{4} \\
\text { 官 }\end{array}$ & 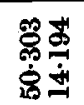 & 声瓷 & 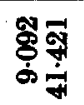 & 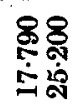 \\
\hline تُ & 日 & $\mathbb{M}$ 买 & $\underset{\infty}{\infty}$ & 35 & 100 & 赵 & 象 & 通 & 5용 & So & $g^{\infty}$ & $\infty \underset{=}{=}$ & \pm 9 \\
\hline & & & & $\infty \infty$ & $\infty 0$ & 어오 & 응으 & 요 & 으음 & @요 & 읖 & $\approx-1$ & $-1=$ \\
\hline$\stackrel{80}{\Sigma}$ & $\begin{array}{l}\infty \\
0 \\
0 \\
0\end{array}$ & 㝴 & 욷욤 & $\stackrel{0}{0}$ & فे & فํ & 5 & 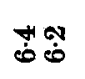 & 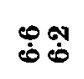 & فำ & 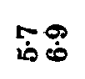 & 40 & فำ \\
\hline 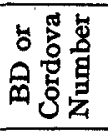 & ద్రీ & స్టేర్తి & \$영 & 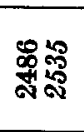 & พิ:ี & 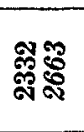 & 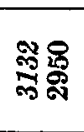 & 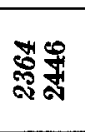 & 융영 & ํㅗㅀ총 & 含哭 & 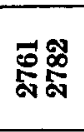 & 켝ㅊ \\
\hline 宽若 & 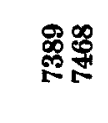 & 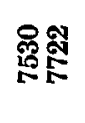 & 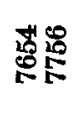 & 足 & 总 & 营是 & 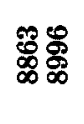 & 串声 & 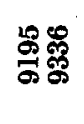 & ్ㅐ욣 & 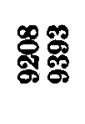 & 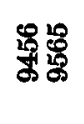 & 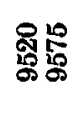 \\
\hline
\end{tabular}




\begin{tabular}{|c|c|c|c|c|c|c|c|c|c|c|c|c|c|}
\hline 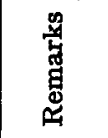 & a 11 & 11 & $\mid \begin{array}{l}\vec{N} \\
\stackrel{\leftrightarrow}{\Xi}\end{array}$ & 11 & 11 & 11 & 11 & 11 & 11 & 11 & 11 & 11 & 11 \\
\hline 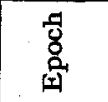 & 吕品 & 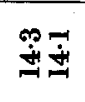 & 両它 & $\stackrel{+}{\sharp}$ & 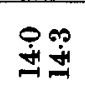 & 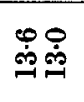 & 菂吊 & 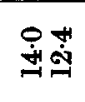 & 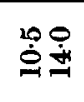 & 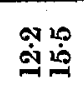 & 岗密 & $\begin{array}{l}\infty \text { L } \\
\dot{\sim} \\
\dot{\sim}\end{array}$ & $\stackrel{4}{=}$ \\
\hline ¿ & - & 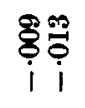 & 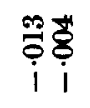 & $\begin{array}{l}\overline{6} \\
\dot{+} \\
\dot{+}\end{array}$ & $\begin{array}{l}5 \\
\dot{8} \\
+\end{array}$ & 11 & 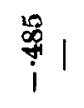 & हैं & $1 \stackrel{?}{+}$ & | ఫ్రి & $\mid \stackrel{\ddot{g}}{i}$ & 11 & $\mid \underset{i}{\stackrel{*}{*}}$ \\
\hline 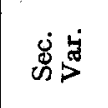 & 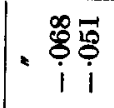 & $\begin{array}{l}\text { 递是 } \\
\stackrel{+}{+}+ \\
\dot{+}\end{array}$ & 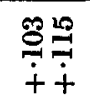 & 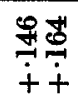 & 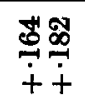 & 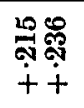 & 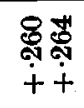 & 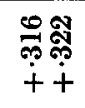 & $\begin{array}{l}\text { 官冚 } \\
\stackrel{+}{+}+\end{array}$ & $\begin{array}{l}\stackrel{\infty}{\forall} \vec{q} \\
\dot{+}+\end{array}$ & 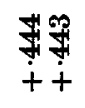 & $\begin{array}{l}\text { 我景 } \\
\dot{+}+\end{array}$ & 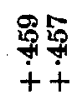 \\
\hline $\begin{array}{l}\dot{8} \\
\text { 点 }\end{array}$ & 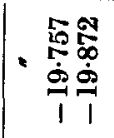 & 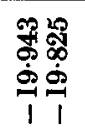 & 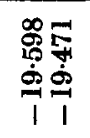 & 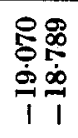 & 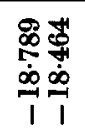 & 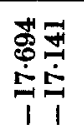 & 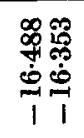 & 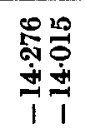 & 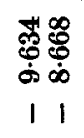 & 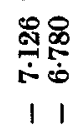 & 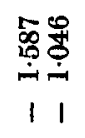 & $\begin{array}{l}8 \\
0 \\
0 \\
0 \\
0 \\
1 \\
1\end{array}$ & 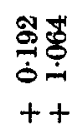 \\
\hline 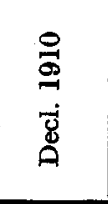 & 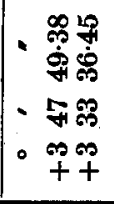 & 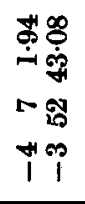 & 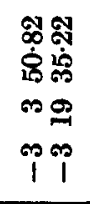 & 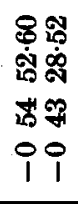 & 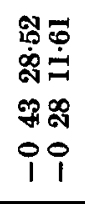 & 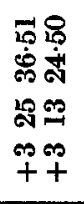 & 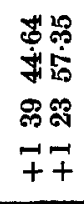 & 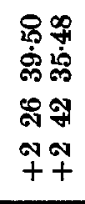 & 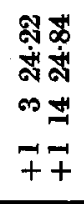 & 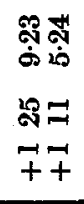 & 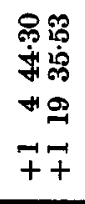 & 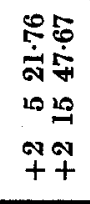 & 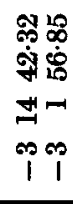 \\
\hline$\neq$ & 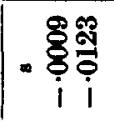 & 范 & $\begin{array}{l}\stackrel{10}{9} \\
\stackrel{9}{9} \\
i\end{array}$ & ह્ઠ & हैं & 11 & 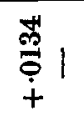 & 营 & $1 \underset{\bar{\sigma}}{\dot{i}}$ & | క్రి & i & 11 & 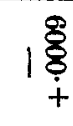 \\
\hline 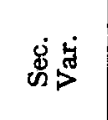 & . ఫ్రి & 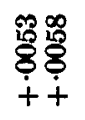 & 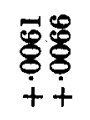 & 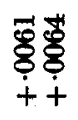 & 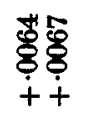 & 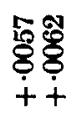 & 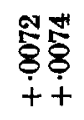 & 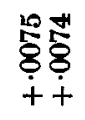 & $\begin{array}{l}\text { 햐야 } \\
\dot{8} \\
\dot{+}\end{array}$ & 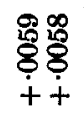 & 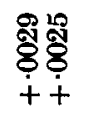 & 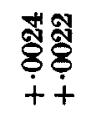 & $\begin{array}{l}\text { 두혀 } \\
\dot{+}+\dot{+}\end{array}$ \\
\hline 造 & 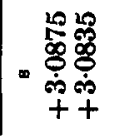 & 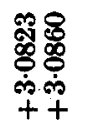 & 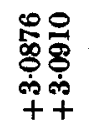 & 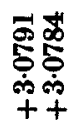 & 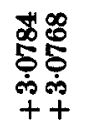 & 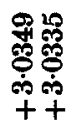 & 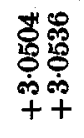 & 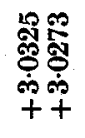 & 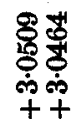 & 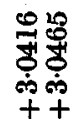 & 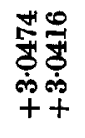 & 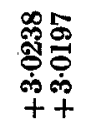 & 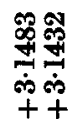 \\
\hline 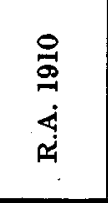 & 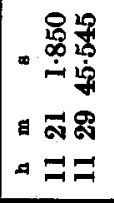 & 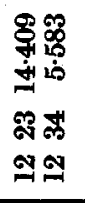 & 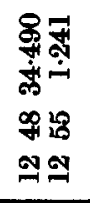 & 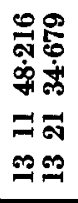 & 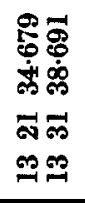 & 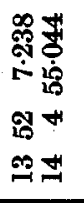 & 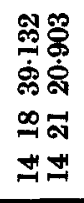 & 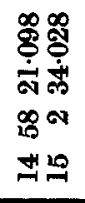 & 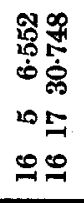 & 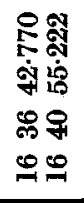 & 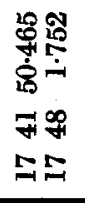 & 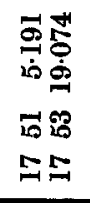 & 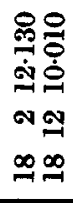 \\
\hline 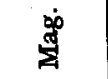 & Di & 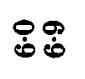 & $\begin{array}{l}\text { No } \\
\text { ô }\end{array}$ & فำ & 입움 & Dִ & 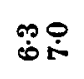 & $\stackrel{\oplus \infty}{+\infty}$ & 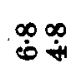 & فㅇํㅇ & 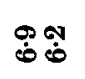 & فे & 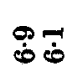 \\
\hline 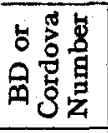 & ฟैํํำ & 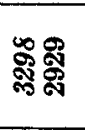 & 周 & 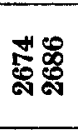 & $\begin{array}{l}\mathscr{0} \\
\text { :영 } \\
\text { 엉 }\end{array}$ & $\begin{array}{l}\text { 范足 } \\
\text { 品 }\end{array}$ & శ్ఞ్ & 串禺 & 承 & 怘哭 & 实商 & 零等 & 领氶 \\
\hline 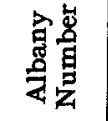 & 영영 & 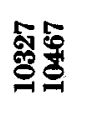 & 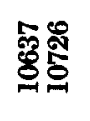 & 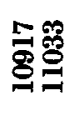 & 营昌 & 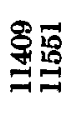 & 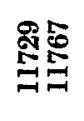 & హే & 带点 & 号芧 & 杽 & 농 & 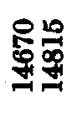 \\
\hline
\end{tabular}




\begin{tabular}{|c|c|c|c|c|c|c|c|c|c|c|c|c|c|}
\hline 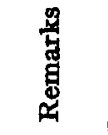 & $\left|\begin{array}{c}\stackrel{\infty}{a} \\
\stackrel{0}{*}\end{array}\right|$ & 11 & $\left.\right|_{\stackrel{\infty}{\infty}} ^{\stackrel{\infty}{\leftrightarrow}}$ & 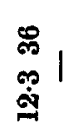 & 11 & 11 & 11 & 11 & $\begin{array}{l}\overline{2} \\
\Re \\
\stackrel{0}{0}\end{array}$ & 11 & 11 & 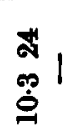 & 11 \\
\hline 遠 & 官离 & 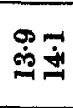 & $\stackrel{\varphi}{=} \underset{ \pm}{ \pm}$ & $\underset{ \pm}{\dot{A}}$ & 富吾 & 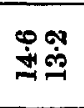 & 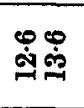 & 亩 & $\begin{array}{l}\text { ஸे下 } \\
\text { ஸे }\end{array}$ & $\stackrel{+}{\dot{g}} \stackrel{9}{二}$ & 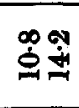 & 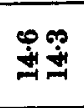 & $\ddot{\infty} \dot{\infty}$ \\
\hline$\because$ & : & i & $1 \frac{8}{8}$ & $\overbrace{i}{ }_{i}$ & 1 ళ్ & 11 & 11 & $\underset{i}{*}$ & 11 & 11 & $\stackrel{\text { ô }}{i} 1$ & $1 \frac{8}{i}$ & 11 \\
\hline 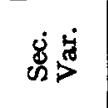 & 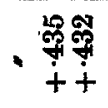 & 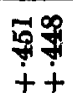 & 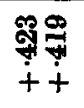 & 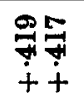 & $\begin{array}{l}\text { 辛 } \\
\stackrel{+}{+} \\
\dot{+}+\end{array}$ & $\begin{array}{l}\text { So̊ } \\
\text { 象 } \\
+\dot{+}\end{array}$ & 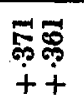 & 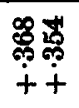 & $\begin{array}{l}\text { 露 } \\
+千+\end{array}$ & 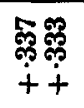 & 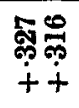 & 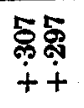 & $\begin{array}{l}\text { 我桇 } \\
+\dot{+}\end{array}$ \\
\hline 通 & 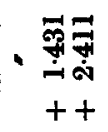 & $\begin{array}{l}\text { \&્木 웡 } \\
\text { के } \\
++\end{array}$ & 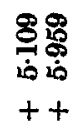 & 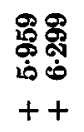 & 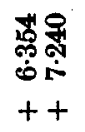 & 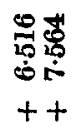 & 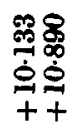 & 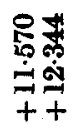 & 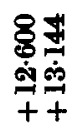 & 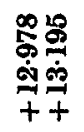 & 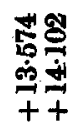 & 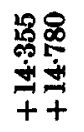 & 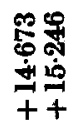 \\
\hline $\begin{array}{l}\stackrel{9}{9} \\
\stackrel{9}{9} \\
\overline{8} \\
\stackrel{8}{9}\end{array}$ & 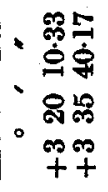 & 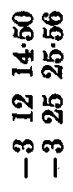 & 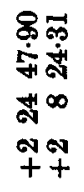 & 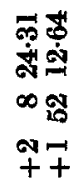 & 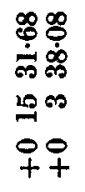 & 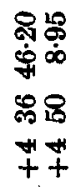 & 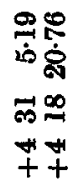 & 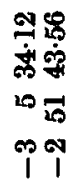 & 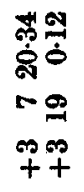 & 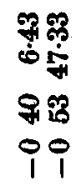 & 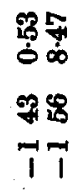 & 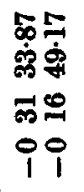 & 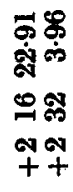 \\
\hline \pm & - $\stackrel{\circ}{8}$ & $\mid \begin{array}{l}\bar{\delta} \\
\dot{+}\end{array}$ & I ఫ్రి & ఫ్రిళ्ठి & 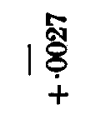 & 11 & 11 & 占各 & 11 & 11 & ఫ్రి & 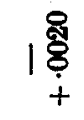 & 11 \\
\hline ॐूँ & . & 这点 & 요ㅇㅛㅛ & $\frac{20}{8} \overline{8}$ & ఫ్రీ & 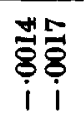 & 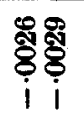 & भิ & 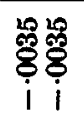 & 予艿 & 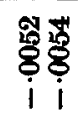 & 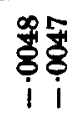 & 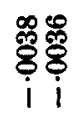 \\
\hline 焉 & 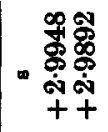 & 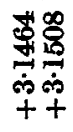 & 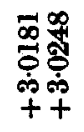 & 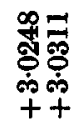 & 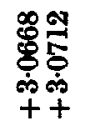 & 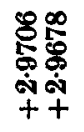 & 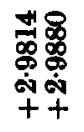 & 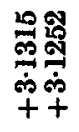 & 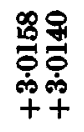 & 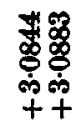 & $\begin{array}{l}\text { क्षे웡 } \\
\text { ஸ் } \\
++\end{array}$ & 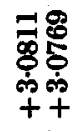 & 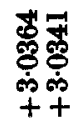 \\
\hline 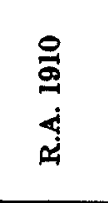 & 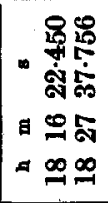 & 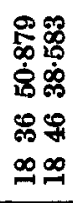 & 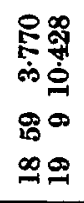 & 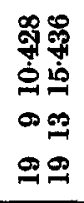 & 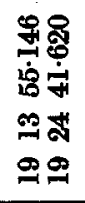 & 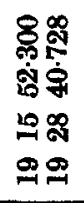 & 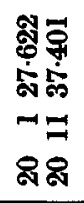 & 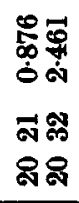 & 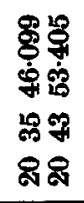 & 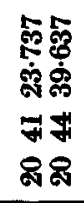 & 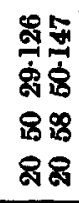 & 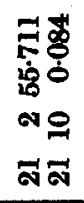 & 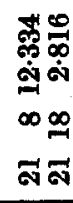 \\
\hline 通 & $\begin{array}{l}900 \\
\dot{H}\end{array}$ & 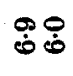 & فํํㄴ & فे & 50 & $\begin{array}{l}00 \\
00 \\
0\end{array}$ & T⿱宀 & فำ & $\begin{array}{l}0 \\
0 \\
0 \\
0\end{array}$ & 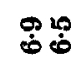 & $\begin{array}{l}00 \\
\dot{0}\end{array}$ & $\begin{array}{ll}\infty & 0 \\
\vdots & 0\end{array}$ & فํํㅇ \\
\hline 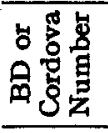 & 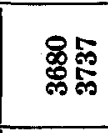 & 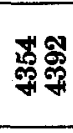 & 㤐品 & స్టైళ & 急 & 동영 & F承总 & \& & 令羿 & 总 용 & 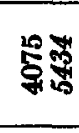 & $\stackrel{8}{\not}$ & 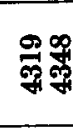 \\
\hline 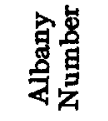 & 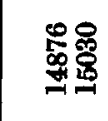 & 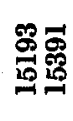 & 总总 & 营蛋 & 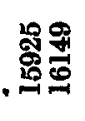 & 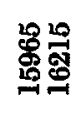 & 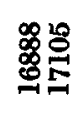 & 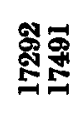 & 旲点 & 苾昂 & 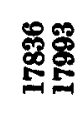 & 串兽 & 点朂 \\
\hline
\end{tabular}




\begin{tabular}{|c|c|c|c|c|c|c|c|c|c|c|c|c|}
\hline 晋 & • & & & 11 & 11 & 11 & 11 & 11 & 11 & 11 & 1 & 1 \\
\hline $\begin{array}{l}8 \\
8 \\
8\end{array}$ & 我思 & 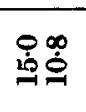 & 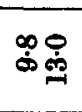 & مَ & 年 & 要 & $\stackrel{\infty}{\infty} \dot{\infty}$ & $\begin{array}{l}\infty \\
\dot{y} \\
\dot{y}\end{array}$ & 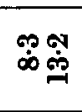 & 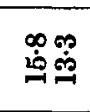 & 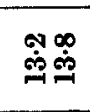 & $\underset{\infty}{\infty} \dot{x}$ \\
\hline 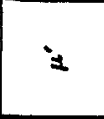 & $\stackrel{\infty}{\circ}+$ & $1 \stackrel{8}{i}$ & 1 & $1 \stackrel{8}{i}$ & 㝘 & I & $1 \stackrel{8}{7}$ & 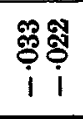 & | & พิ⿱宀⿻⺀大 & 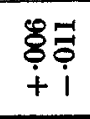 & $1 \stackrel{\text { है }}{\dot{t}}$ \\
\hline 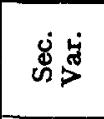 & 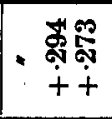 & 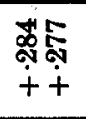 & 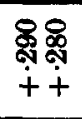 & 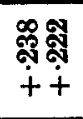 & 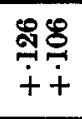 & 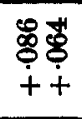 & 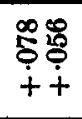 & 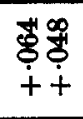 & 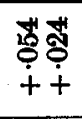 & 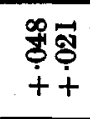 & 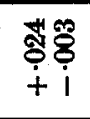 & $\overbrace{i}^{\text {ț }}$ \\
\hline 悹 & 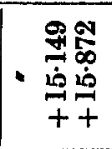 & 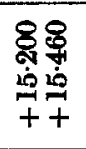 & 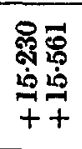 & 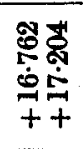 & 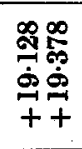 & 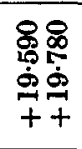 & 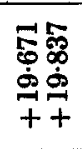 & 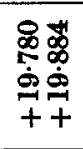 & 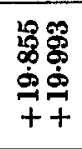 & 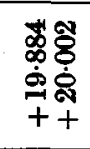 & 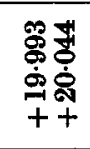 & 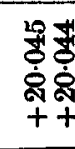 \\
\hline 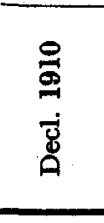 & 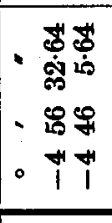 & 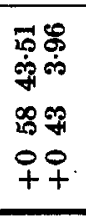 & 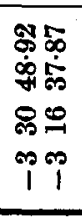 & 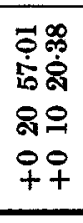 & 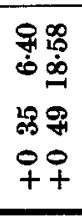 & 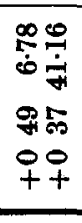 & 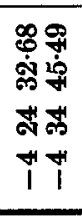 & 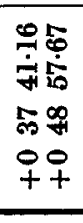 & 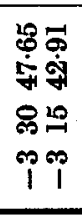 & 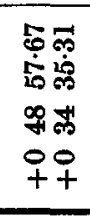 & 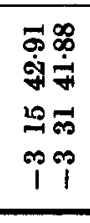 & $\frac{0}{90}$ \\
\hline \pm & i & 18 & 11 & $\mid \stackrel{\tilde{8}}{\dot{+}}$ & & 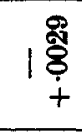 & $\mid \stackrel{\vec{j}}{\overrightarrow{+}}$ & 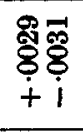 & $\mid$ & 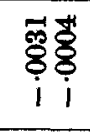 & 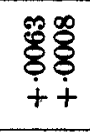 & $\mid \stackrel{\infty}{8}$ \\
\hline 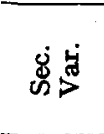 & 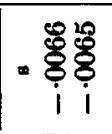 & 突实 & 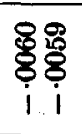 & 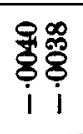 & 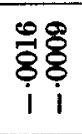 & 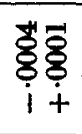 & 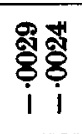 & $\begin{array}{l}\widetilde{\delta} \delta \\
\dot{\delta} \\
+ \\
+\end{array}$ & 둥ㅇㅇ & 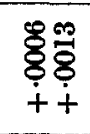 & ర్ઠిં & ఫ్రిక్రి \\
\hline 总 & 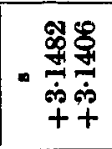 & 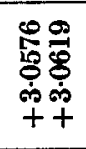 & 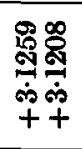 & 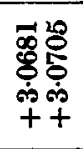 & 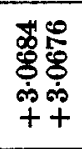 & 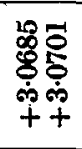 & 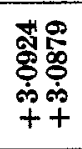 & 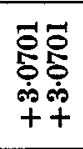 & 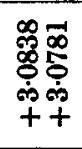 & 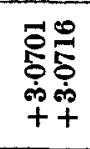 & 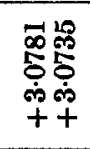 & 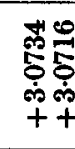 \\
\hline 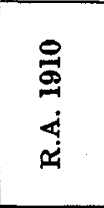 & 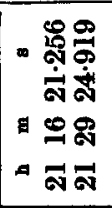 & 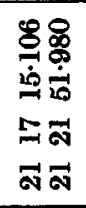 & 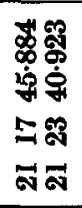 & 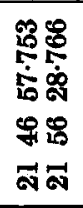 & 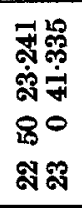 & 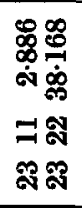 & 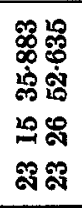 & 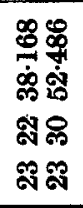 & 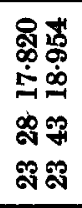 & 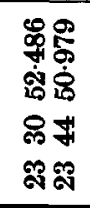 & 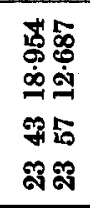 & 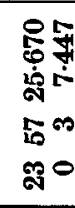 \\
\hline 离 & $\begin{array}{l}\dot{0} \\
\dot{\phi} \dot{0}\end{array}$ & & & 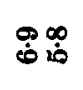 & தُ & $\stackrel{\infty}{\dot{0}}$ & 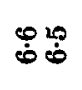 & ザ & فํ. & $\begin{array}{l}\varphi \infty \\
\dot{0}\end{array}$ & 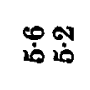 & $\stackrel{\infty}{\infty}$ \\
\hline 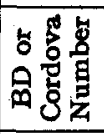 & 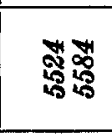 & 荤密 & $\stackrel{\infty}{\infty}$ & $\begin{array}{l}\text { कo: } \\
\text { क्ञ⿰氵 }\end{array}$ & 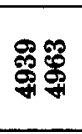 & 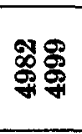 & 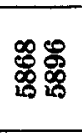 & 象弯总 & 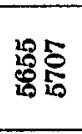 & 常范 & 它蛋 & $\stackrel{8}{5}^{\circ}$ \\
\hline 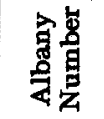 & 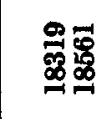 & 裿点 & 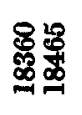 & 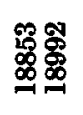 & 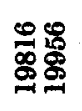 & 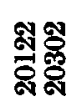 & 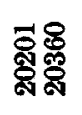 & 萞 & 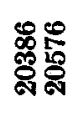 & స్ㅊ용 & 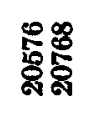 & \\
\hline
\end{tabular}

